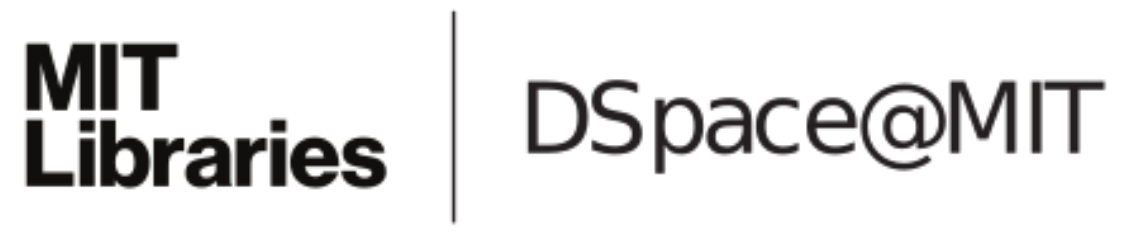

\author{
MIT Open Access Articles
}

In-scene LWIR downwelling radiance estimation

The MIT Faculty has made this article openly available. Please share how this access benefits you. Your story matters.

Citation: Pieper, M., D. Manolakis, E. Truslow, T. Cooley, M. Brueggeman, A. Weisner, and J. Jacobson. "In-Scene LWIR Downwelling Radiance Estimation." Edited by John F. Silny and Emmett J. lentilucci. Imaging Spectrometry XXI (September 19, 2016).

As Published: http://dx.doi.org/10.1117/12.2239138

Publisher: SPIE

Persistent URL: http://hdl.handle.net/1721.1/116580

Version: Final published version: final published article, as it appeared in a journal, conference proceedings, or other formally published context

Terms of Use: Article is made available in accordance with the publisher's policy and may be subject to US copyright law. Please refer to the publisher's site for terms of use. 


\section{In-scene LWIR downwelling radiance estimation}

M. Pieper, D. Manolakis, E. Truslow, T. Cooley, M. Brueggeman, et al.

M. Pieper, D. Manolakis, E. Truslow, T. Cooley, M. Brueggeman, A. Weisner, J. Jacobson, "In-scene LWIR downwelling radiance estimation," Proc. SPIE 9976, Imaging Spectrometry XXI, 99760E (19 September 2016); doi: $10.1117 / 12.2239138$

SPIE Event: SPIE Optical Engineering + Applications, 2016, San Diego, California, United States 


\title{
In-Scene LWIR Downwelling Radiance Estimation
}

\author{
M. Pieper ${ }^{a}$, D. Manolakis ${ }^{b}$, E. Truslow ${ }^{b}$, T. Cooley ${ }^{c}$, M. Brueggeman $^{d}$, A. Weisner ${ }^{d}$, and \\ J. Jacobson ${ }^{d}$ \\ ${ }^{a}$ Northeastern University, 360 Huntington Ave, Boston, MA 02115 \\ ${ }^{b}$ MIT Lincoln Laboratory, 244 Wood Street, Lexington, MA 02420 \\ ${ }^{c}$ Space Vehicles Directorate Air Force Research Laboratory, 2251 Maxwell Avenue, Kirtland \\ AFB, NM 87117 \\ ${ }^{d}$ National Air and Space Intelligence Center, Wright-Patterson AFB, OH 45433
}

\begin{abstract}
Effective hyperspectral thermal infrared imaging requires accurate atmospheric compensation to convert the measured at-sensor radiance to the ground radiance. The ground radiance consists of the thermal emission of the material and the reflected downwelling radiance. An accurate estimate of the downwelling radiance is required for temperature-emissivity separation (TES) to remove the spectrally sharp reflected atmospheric effects and retrieve a smooth and accurate material emissivity to use for detection.

Determination of the downwelling radiance is difficult due to the fact that a down-looking sensor has no knowledge of the atmospheric properties above its line of sight. As the sensor altitude increases and more of the atmospheric emitters are below the sensor, a relationship forms between the upwelling and downwelling radiances. This relationship comes at the expense of increased pixel size, which increases the likelihood of mixed pixels and nonlinear spectral mixing.

In this paper improvements to methods used to estimate the downwelling radiance of low altitude collections are proposed. The ground radiances of reflective pixels are used to estimate the atmosphere above the sensor. The reflective pixels are identified from their sharp atmospheric spectral features. Using the assumption that emissivity spectra are smooth across the narrow reflected atmospheric downwelling radiance features, the temperatures and emissivities are then separated for the reflective pixels using a look-up-table of downwelling radiances. The downwelling radiance that provides the best overall fit for the reflective pixels is then chosen as the scene downwelling radiance.
\end{abstract}

\section{INTRODUCTION}

A hyperspectral imaging (HSI) sensor measures the electromagnetic spectrum at hundreds of narrow contiguous wavelength bands, allowing for fine spectral measurements. These fine spectral measurements are combined to form a spectrum at each pixel with $N$ spectral bands [1]. The spectrum is then used to identify materials on the ground which have their own unique spectral properties. Long wave infrared (LWIR) hyperspectral imaging sensors measure thermal radiation between $8 \mu \mathrm{m}$ to $14 \mu \mathrm{m}$.

The material properties of interest are a material's emissivity and temperature. A material library typically consists of emissivity spectra of interest, however the the data spectra consist of measured at-sensor radiance spectra. In order to compare the two, the measured at-sensor radiance needs to be converted to emissivity. The sensor radiance is converted to ground radiance by compensating for atmospheric effects on the ground radiance over its path to the sensor. Atmospheric compensation requires accurate knowledge of the atmospheric transmission and upwelling radiance at each of the $\mathrm{N}$ spectral bands. The ground radiance consists of the thermal emission of the material and the reflected downwelling radiance [2]. The thermal emission component includes the material properties of interest. With an accurate estimate of the downwelling radiance, the reflected

This work is sponsored by the Air Force Research Laboratory under Air Force Contract \#FA8721-05-C-0002. Opinions, interpretations, conclusions, and recommendations are those of the author and are not necessarily endorsed by the United States Government.

Imaging Spectrometry XXI, edited by John F. Silny, Emmett J. lentilucci, Proc. of SPIE Vol. 9976, 99760E · @ 2016 SPIE · CCC code: 0277-786X/16/\$18 - doi: 10.1117/12.2239138 
component can be removed, and the thermal emission can be separated into temperature and emissivity using a technique known as temperature emissivity separation (TES) [3]. The emissivity spectrum can then be used to determine the materials within the pixel

In-scene atmospheric compensation (ISAC) algorithms exist that use in-scene pixels that contain blackbody like materials to determine the atmospheric transmission and upwelling radiance [2]. Similar assumptions cannot be used to determine the downwelling radiance because only pixels containing materials with low unknown emissivities contain a reflected downwelling radiance component. In addition, the downwelling radiance consists of the entire atmosphere, and the down-looking sensor has no knowledge of the atmospheric properties above the sensor. The estimated atmospheric transmission and upwelling radiance only give knowledge of the atmosphere below the sensor. Due to the lack of knowledge above the sensor, a look-up-table of (LUT) of outside models for the downwelling radiance needs to be utilized [3].

ISAC is done on a scene to convert the at-sensor radiance spectrum of each pixel to ground radiance. Low emissivity ground radiance spectra are then found that differ from blackbodies and contain a downwelling radiance component. At this stage, the number of unknowns is $2 N+1$ for each pixel spectrum. $N$ unknowns for each downwelling radiance and emissivity spectrum and 1 for the temperature. The atmosphere is assumed to be homogenous throughout a scene, so the downwelling radiance spectrum remains constant for all the pixel spectra. Current algorithms work by doing TES on the low emissivity spectra using each of the downwelling radiance models, . The downwelling radiance model that supplies the best overall TES results is chosen as the downwelling radiance of the scene [3].

In order to determine the correct emissivity spectra, the correct downwelling radiance model needs to be determined. Small errors in the downwelling radiance estimation can effect the temperature and shape of the emissivity spectra. Large errors will cause TES to completely fail and give unphysical results. In this paper, techniques to improve estimation of the in-scene downwelling radiance are developed. The affects of downwelling radiance estimate errors on TES are studied, and whether the current technique gives the correct unique solution. The paper is organized as follows. Background descriptions of the LWIR atmospheric model, atmospheric compensation, and TES are given in section 2. The method used to estimate the downwelling radiance and improvements are described in section 3. The procedure used to test the estimation method is discussed in section 4 . The results are shown and discussed in section 5. A summary is provided in section 6 .

\section{BACKGROUND}

A LWIR hyperspectral remote sensing environment has three main components as shown in Fig. 1:

- Ground Radiance

The ground radiance consists of the emitted thermal radiation from materials on the ground and the reflected radiation from the atmosphere. The ground radiance spectrum of a pixel is modeled as [2]

$$
L_{\mathrm{G}}\left(\lambda_{k}\right)=\epsilon\left(\lambda_{k}\right) B\left(\lambda_{k} ; T_{\mathrm{G}}\right)+\left[1-\epsilon\left(\lambda_{k}\right)\right] L_{\mathrm{D}}\left(\lambda_{k}\right)
$$

where $\lambda_{k}$ is the wavelength of a spectral band. The ground emission is $\epsilon\left(\lambda_{k}\right) B\left(\lambda_{k} ; T_{\mathrm{G}}\right)$ where $\epsilon\left(\lambda_{k}\right)$ is the surface emissivity spectrum of the material and $T_{\mathrm{G}}$ is the ground temperature. $B\left(\lambda_{k} ; T_{\mathrm{G}}\right)$ is the Planck radiation function for a blackbody at the ground temperature

$$
B\left(\lambda ; T_{\mathrm{G}}\right)=\frac{c_{1}}{\lambda_{k}^{5}\left[\exp \left(c_{2} /\left(\lambda_{k} T_{\mathrm{G}}\right)\right)-1\right]}
$$

where $c_{1}=1.19104 \times 10\left(\mu \mathrm{W} / \mathrm{cm}^{2} / \mathrm{sr} / \mu \mathrm{m}\right)-\mu \mathrm{m}^{5}$ and $c_{2}=14387.7 \mu \mathrm{m}-\mathrm{K}$. The ground emission is the most important component because it contains the spectral emissivity properties of the ground material and its temperature. The reflected radiation is $\left[1-\epsilon\left(\lambda_{k}\right)\right] L_{\mathrm{D}}\left(\lambda_{k}\right)$, where the reflectance is $\left[1-\epsilon\left(\lambda_{k}\right)\right]$, and $L_{\mathrm{D}}\left(\lambda_{k}\right)$ is the hemispherical downwelling radiance from the atmosphere. 


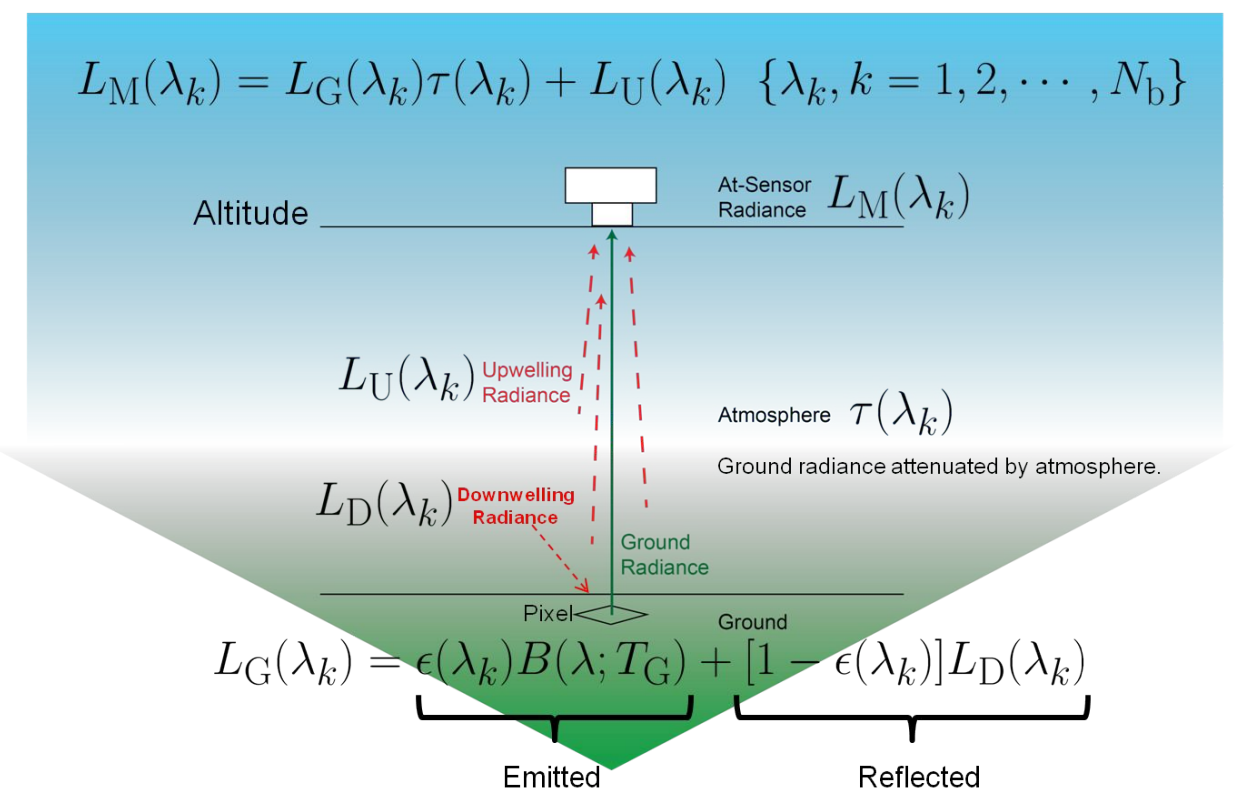

Figure 1. Illustration of the LWIR hyperspectral remote sensing environment.

\section{- Atmosphere}

As the ground radiance travels up toward the sensor, it is affected by the atmosphere. The atmosphere absorbs and emits radiation. The atmospheric transmission $\tau\left(\lambda_{k}\right)$, upwelling radiance $L_{\mathrm{U}}\left(\lambda_{k}\right)$, and downwelling radiance $L_{\mathrm{D}}\left(\lambda_{k}\right)$ depend on the types of gases present in the atmosphere and their concentrations with altitude. An atmospheric gas profile describes how the concentration of the gas changes with altitude. The atmosphere is approximated to be homogeneous throughout a scene. Fig. 2 shows an example of an atmospheric TUD (for Transmission, Up-welling, Downwelling radiance) model at a sensor altitude of 1.5 $\mathrm{km}$.

The atmospheric transmission in the LWIR region between $8-13 \mu \mathrm{m}$ is quite high, and is why the hyperspectral sensor is designed to measure radiation inside this atmospheric window. Other benefits include the ability to measure pixel spectra at night, and the lack of aerosols and scattering. Just outside this region the transmission quickly drops due to water absorption. The main atmospheric absorption gases in the LWIR window region are $\mathrm{H}_{2} \mathrm{O}, \mathrm{O}_{3}$, and $\mathrm{CO}_{2}$. The molecular properties of the gases determine at which wavelengths they absorb and emit radiation. There are several significant gas absorption features within the window region. There are $\mathrm{H}_{2} \mathrm{O}$ absorption features between $11.62-11.84 \mu \mathrm{m}$ and $12.2-12.7 \mu \mathrm{m}$. The $\mathrm{H}_{2} \mathrm{O}$ profile typically has a high concentration at the surface and decreases till about $10 \mathrm{~km}$. The concentration of $\mathrm{H}_{2} \mathrm{O}$ between scenes is more variable compared to the other atmospheric gases. The main $\mathrm{O}_{3}$ absorption feature occurs between 9.4-9.84 $\mu \mathrm{m}$. There are insignificant amounts of $\mathrm{O}_{3}$ till about $10 \mathrm{~km}$. The depth of these absorption features describe the total amount of the gases in the atmosphere. A majority of the large absorption effects of the gases are avoided at the spectral bands near 8.38, 8.85, 10.07, 10.4, 11.13, 11.4, and $12.2 \mu \mathrm{m}$ as shown in Fig. 2.

The atmospheric transmission and emission are related by $1-\tau \approx \epsilon$. When the transmittance is low, the atmospheric absorption is high, which makes the upwelling radiance emission high. While the upwelling radiance consists only of emission from gases between the sensor and the ground, the downwelling radiance $L_{\mathrm{D}}\left(\lambda_{k}\right)$ consists of the entire atmosphere [2]. For this reason, the downwelling radiance is greater than the upwelling radiance, and the two become more similar as the altitude of the sensor increases. When the sensor is below $10 \mathrm{~km}$, the sensor will be below the $\mathrm{O}_{3}$ and the upwelling radiance will not include the emission from the $\mathrm{O}_{3}$. However, the downwelling radiance which includes the entire atmosphere will include the $\mathrm{O}_{3}$ emission. The difference between $\mathrm{O}_{3}$ emission in $L_{\mathrm{U}}\left(\lambda_{k}\right)$ and $L_{\mathrm{D}}\left(\lambda_{k}\right)$ at low sensor altitudes between 9.4-9.84 $\mu \mathrm{m}$ is shown in Fig. 2. 


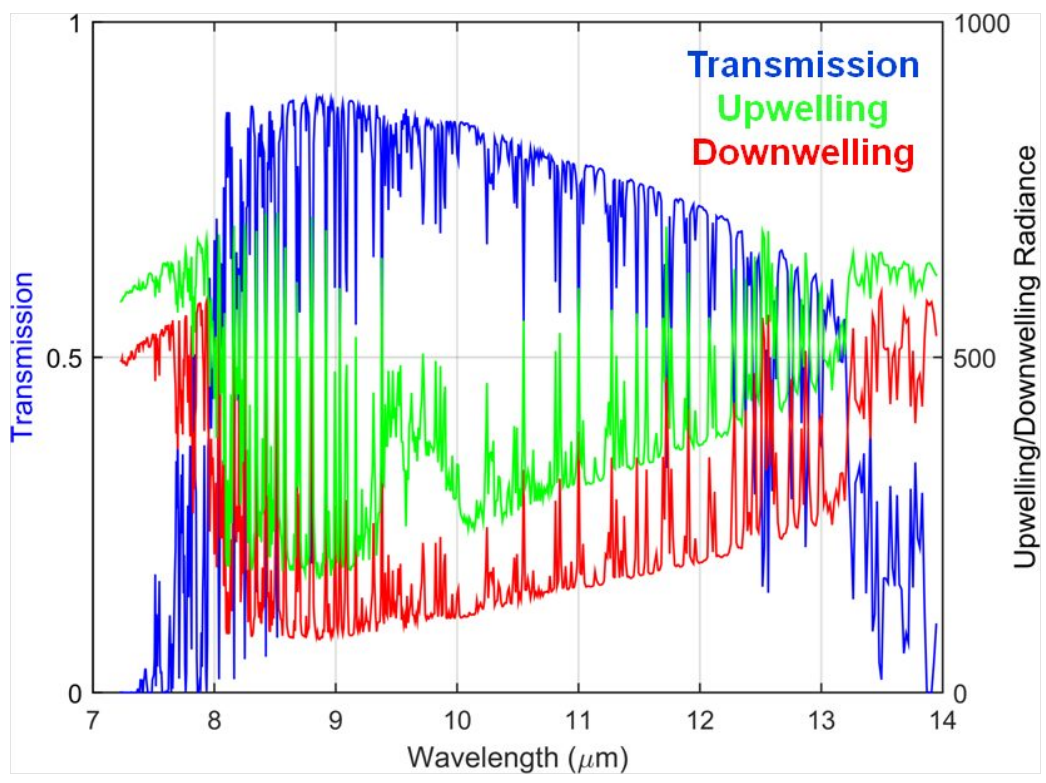

Figure 2. Atmospheric TUD Model.

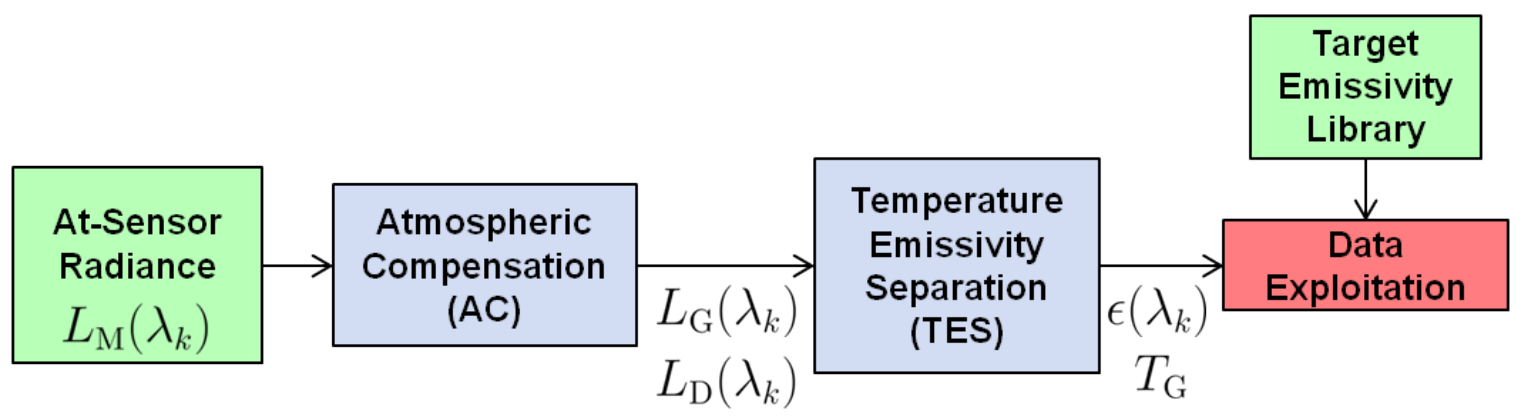

Figure 3. Hyperspectral Imaging Processing Pipeline

- Measured At-Aperture Radiance

The measured at-aperture radiance spectrum consists of ground radiance spectrum effected by the atmosphere, and is modeled as [2]

$$
L_{\mathrm{M}}\left(\lambda_{k}\right)=\epsilon\left(\lambda_{k}\right) B\left(\lambda_{k} ; T_{\mathrm{G}}\right) \tau\left(\lambda_{k}\right)+\left[1-\epsilon\left(\lambda_{k}\right)\right] L_{\mathrm{D}}\left(\lambda_{k}\right) \tau\left(\lambda_{k}\right)+L_{\mathrm{U}}\left(\lambda_{k}\right)=L_{\mathrm{G}}\left(\lambda_{k}\right) \tau\left(\lambda_{k}\right)+L_{\mathrm{U}}\left(\lambda_{k}\right)
$$

The main goal of a HSI remote sensing system is to study the materials on the ground. This is typically done in the emissivity domain where the emissivity spectra of pixels are compared to a target emissivity library. The target emissivity library contains lab measured emissivity spectra for materials of interest. In order to make this comparison, the ground emissivity must be extracted from the measured at-aperture radiance. Fig. 3 shows a typical HSI processing pipeline, where the ground emissivity and temperature are extracted from the measured radiance. The processing from measured radiance to ground emissivity consists of atmospheric compensation followed by temperature emissivity separation, which are discussed in the following subsections.

\subsection{Atmospheric Compensation (AC)}

$\mathrm{AC}$ is the process where atmospheric effects are removed from the measured radiance to retrieve the ground radiance, described in equation (1). To remove the atmospheric effects, a TUD atmospheric model is estimated for the entire scene. With an accurate estimate of the atmospheric transmission $\tau\left(\lambda_{k}\right)$ and upwelling radiance $L_{\mathrm{U}}\left(\lambda_{k}\right)$, transmission losses and emission gains from the atmosphere can be compensated to find the ground 
radiance $L_{\mathrm{G}}\left(\lambda_{k}\right)$. Rearranging Eq. 3 we find that

$$
L_{\mathrm{G}}\left(\lambda_{k}\right)=\frac{L_{\mathrm{M}}\left(\lambda_{k}\right)-L_{\mathrm{U}}\left(\lambda_{k}\right)}{\tau\left(\lambda_{k}\right)}
$$

In-scene Atmospheric Compensation (ISAC) is a method where the transmission and upwelling radiance of a scene's atmosphere are estimated using natural blackbody or near blackbody materials of varying temperatures that are present in the scene. Multiple versions with varying methods of selecting blackbody pixels exist. The method discussed here is from [2]. Pixels that contain materials such as water and vegetation have $\epsilon \approx 1$ and are key candidates for blackbody like pixels, as shown in Fig. 10a. The quality of ISAC is determined by how well the in-scene blackbody like pixels resemble actual blackbodies. When $\epsilon \approx 1$ the measured radiance in Eq. 3 simplifies to

$$
L_{\mathrm{M}}\left(\lambda_{k}\right) \cong \epsilon\left(\lambda_{k}\right) B\left(\lambda_{k} ; T_{\mathrm{G}}\right) \tau\left(\lambda_{k}\right)+L_{\mathrm{U}}\left(\lambda_{k}\right)
$$

Since the downwelling radiance term $[1-\epsilon] L_{\mathrm{D}} \approx 0$. The simplified measured radiance equation is a linear function for each wavelength band, of the independent variable $B\left(\lambda_{k} ; T_{\mathrm{G}}\right)$, where $\tau\left(\lambda_{k}\right)$ and $L_{\mathrm{U}}\left(\lambda_{k}\right)$ are the slope and y-intercept. The difficulty of ISAC lies in the determining which of the pixels in the scene are close to being blackbodies, and what are their corresponding temperatures.

The ground temperature estimate $\hat{T}_{\mathrm{G}}$ of each pixel is found by finding the maximum band brightness temperature in each pixel spectrum. The band brightness temperature is the inverse of the Planck function $B\left(\lambda_{k} ; T_{\mathrm{G}}\right)$ and is the temperature at which a blackbody emits radiance $L$ at wavelength $\lambda_{k}$ and is defined as

$$
T_{B}\left(\lambda_{k} ; L\right)=\frac{c_{2}}{\lambda \ln \left(c_{1} /\left(\lambda_{k}^{5} L\right)+1\right)}=B^{-1}\left(\lambda_{k} ; L\right)
$$

The band that has the most maximum pixel brightness temperatures is chosen as the reference band $\lambda_{R}$, and only the pixels that share this band as their maximum are kept for further searching of possible blackbodies. The reference band will typically be at an atmospherically clear wavelength.

A blackbody spectrum $B\left(\lambda ; \hat{T}_{\mathrm{G}}\right)$ is generated with Eq. 2 over the entire spectral range of the sensor for each pixel using the maximum brightness temperature as the ground temperature estimate. These spectra represent the ground radiance of the possible blackbody pixels. Using the blackbody spectra as a ground radiance reference, scatter plots are created of $B\left(\lambda_{k} ; T_{\mathrm{G}}\right)$ vs. $L_{\mathrm{M}}\left(\lambda_{k}\right)$ for each band. An example of a $B\left(\lambda_{k} ; T_{\mathrm{G}}\right)$ vs. $L_{\mathrm{M}}\left(\lambda_{k}\right)$ scatter plot for the $8.87 \mu \mathrm{m}$ band is shown in Fig. 4. These plots show how the possible ground radiance compares to the measured radiance for the pixels at each band. The overall trend of each scatter plot is determined by the transmission and upwelling radiance at the corresponding band as described by Eq. 5 . At a constant $B\left(\lambda_{k} ; T_{\mathrm{G}}\right)$ the value of a pixel's $L_{\mathrm{M}}\left(\lambda_{k}\right)$ is determined by the pixel's $\epsilon\left(\lambda_{k}\right)$. The pixels at the top of the scatter plot have the highest emissivity and are more likely to be blackbody like pixels. By fitting a line to the likely blackbody like pixels in each band scatter plot, coefficients proportional to o $\tau\left(\lambda_{k}\right)$ and $L_{\mathrm{U}}\left(\lambda_{k}\right)$ are found.

\subsection{Temperature Emissivity Separation (TES)}

The ground radiance consists of the emitted radiation from materials on the ground and the reflected radiation from the atmosphere. The spectral emissivity features of solids are wide compared to the sharp spectral features of atmospheric gases [3]. When observing a reflective ground material, one sees sharp features in $L_{\mathrm{G}}\left(\lambda_{k}\right)$ from the gas emissions of the atmosphere. In order to determine the emissivity of the ground material, these sharp atmospheric features need to be removed. However, the magnitude of the features are dependent on the emissivity itself. TES removes the reflected radiation component, and separates the temperature and emissivity from the emission component.

With an accurate estimate of $L_{\mathrm{D}}\left(\lambda_{k}\right)$ the emissivity can be calculated for a given temperature from Eq.

$$
\hat{\epsilon}\left(\lambda_{k}\right)=\frac{L_{\mathrm{G}}\left(\lambda_{k}\right)-L_{\mathrm{D}}\left(\lambda_{k}\right)}{B\left(\lambda_{k} ; \hat{T}_{\mathrm{G}}\right)-L_{\mathrm{D}}\left(\lambda_{k}\right)}
$$

where $\hat{\epsilon}\left(\lambda_{k}\right)$ and $\hat{T}_{\mathrm{G}}$ are estimates of the ground emissivity and temperature respectively. When the estimated ground temperature is different from the actual temperature, the reflected atmospheric features are visible in 


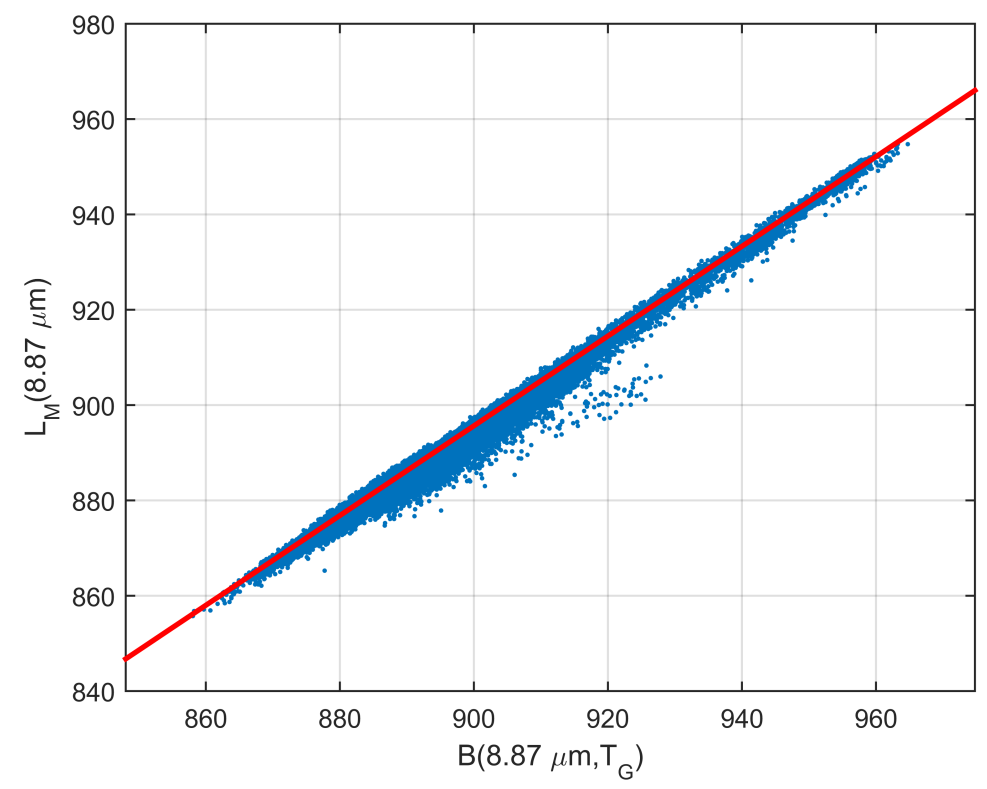

Figure 4. $B\left(\lambda_{k} ; T_{\mathrm{G}}\right)$ vs. $L_{\mathrm{M}}\left(\lambda_{k}\right)$ scatter plot for $8.87 \mu \mathrm{m}$ band.

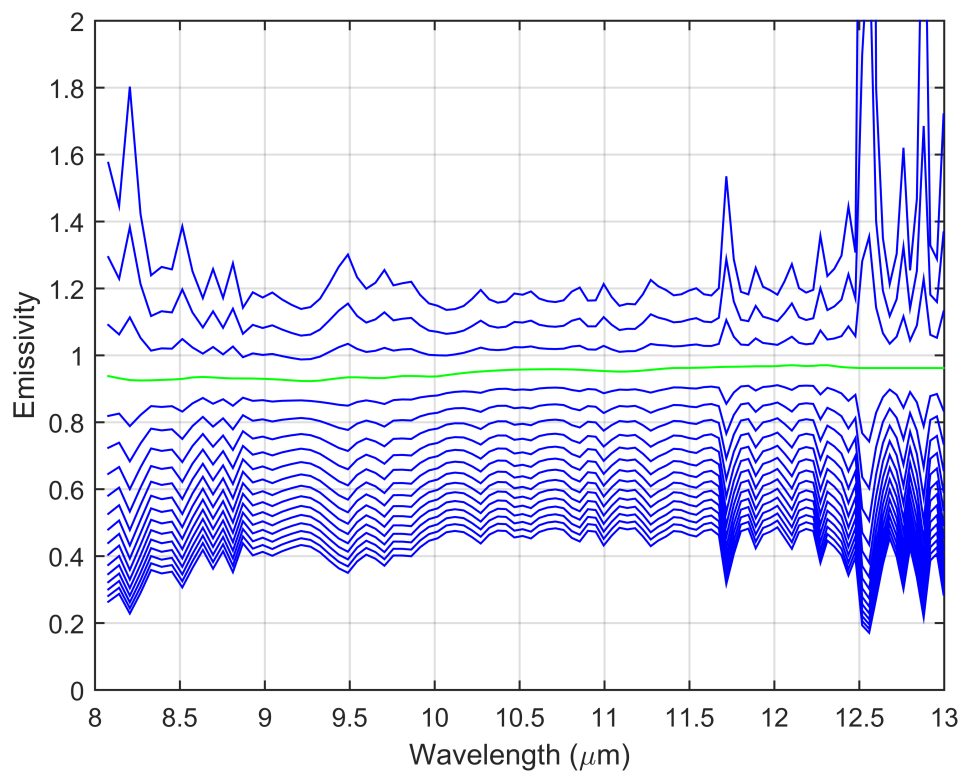

Figure 5. Emissivity estimates at varying temperatures. The green emissivity is the correct temperature.

the estimated emissivity. As the estimated ground temperature approaches the actual temperature, the reflected features in the estimated emissivity decrease in size [3]. Fig. 5 shows the emissivity estimate for varying temperatures. When the temperature estimate is off, the water and ozone emission from the downwelling radiance are clearly visible. When the temperature is correct, the smooth green emissivity spectrum is found.

Temperature emissivity separation is a numerical optimization problem where the ground temperature estimate $\hat{T}_{\mathrm{G}}$ that provides the smoothest emissivity spectrum is determined [3]. The maximum brightness temperature of the ground radiance is used as the initial $\hat{T}_{\mathrm{G}}$. Using the current $\hat{T}_{\mathrm{G}}$ an emissivity estimate is calculated, and smoothed using N-band boxcar averaging to obtain $\bar{\epsilon}$. A new ground radiance is calculated using the smoothed emissivity spectrum $\bar{\epsilon}$ so that

$$
\overline{L_{\mathrm{G}}}\left(\lambda_{k}\right)=\bar{\epsilon}\left(\lambda_{k}\right) B\left(\lambda_{k} ; \hat{T}_{\mathrm{G}}\right)+\left(1-\bar{\epsilon}\left(\lambda_{k}\right)\right) L_{\mathrm{D}}\left(\lambda_{k}\right)
$$


The error between the actual and calculated ground radiances is defined as

$$
E=\sqrt{\frac{1}{k_{2}-k_{1}+1} \sum_{k_{1}}^{k_{2}}\left[L_{\mathrm{G}}\left(\lambda_{k}\right)-\overline{L_{\mathrm{G}}}\left(\lambda_{k}\right)\right]^{2}}
$$

where $k_{1}$ and $k_{2}$ are a range of spectral bands. The ground temperature estimate is updated till a $\hat{T}_{\mathrm{G}}$ is found that provides the minimum $E$. This minimum occurs at the correct $\hat{T}_{\mathrm{G}}$, when the calculated emissivity is the smoothest, and the smoothing error is minimal.

\subsection{Downwelling Radiance}

The accuracy of TES is determined by the quality of the AC used to determine $L_{\mathrm{G}}$ and the quality of the estimated $L_{\mathrm{D}}$. Inaccuracies in the $\mathrm{AC}$ and the estimated $L_{\mathrm{D}}$ can prevent TES from finding a temperature that removes the sharp reflected atmospheric features, and can effect the temperature and the shape of the emissivity spectrum.

The downwelling radiance is the most difficult atmospheric component to estimate due to the lack of in-scene evidence. It consists of the entire atmosphere, including the unmeasured portion above the sensor. In-scene evidence of the downwelling radiance comes from the upwelling radiance and reflective pixels present in the scene [3]. The upwelling and downwelling radiance are related over the portion of the atmosphere that the two overlap. The relationship between the two strengthens as the altitude of the sensor increases. Evidence of the downwelling radiance for the entire atmosphere is provided as sharp reflected gas features within the ground radiance spectra of pixels with low $\epsilon$

Temperature emissivity separation and estimation of the downwelling radiance are linked. The amount of downwelling radiance in the reflective pixels is dependent on the unknown emissivity spectra of the pixels. TES is done on the reflective ground radiances using an estimated downwelling radiance. The quality of the estimated downwelling radiance is determined by the TES quality of the determined emissivity spectra [3].

\section{DOWNWELLING RADIANCE ESTIMATION}

Combined AC and TES requires the estimation of at least $4 N+1$ parameters where $N$ is the number of spectral bands of the sensor[4]. Due to the large dimensionality of the problem, attempting to estimate all the parameters at once may lead to a non-unique solution that provides believable smooth but incorrect emissivity spectra. To limit the dimensionality of the problem, the transmission and upwelling radiance based on in-scene evidence from ISAC are used for AC to convert a scene to ground radiance and reduce the number of unknowns to $2 N+1$. The downwelling radiance is then estimated from just the ground radiance of reflective pixels using the algorithm shown in Fig. 6.

Reflective pixels are pixels that contain low emissivity materials and contain a reflected downwelling component in their ground radiance spectra. Examples of low emissivity spectra and simulated ground radiance spectra are shown in Fig 10. Reflective pixels are pixels that least resemble blackbodies. They can be found in a scene by calculating the brightness temperatures of the ground radiance spectra in a scene, and finding the pixels with the largest brightness temperature variance. The brightness temperature variability will be effected by the variance of the emissivity and its magnitude, along with the features of the downwelling radiance.

Since the emissivity is unknown for reflective pixels, the downwelling radiance cannot be directly estimated from a scene. Due to the lack of in-scene evidence, an accurate atmospheric model is needed to determine the downwelling radiance. The atmospheric model should contain atmospheric temperature and gas profiles that closely match those of the scene for the entire height of the atmosphere.

Atmospheric models for the downwellling radiance are generated using MODTRAN (MODerate resolution atmospheric TRANsmission), a radiative transfer algorithm [5]. The inputs to MODTRAN consist of atmospheric and sensor parameters. The atmospheric parameters consist of temperature and gas profiles that describe the temperature and gas concentrations of the atmosphere with altitude. Scaling parameters are used to finely adjust the concentration of the gases. The sensor parameters include sensor altitude, latitude, longitude, wavelength 


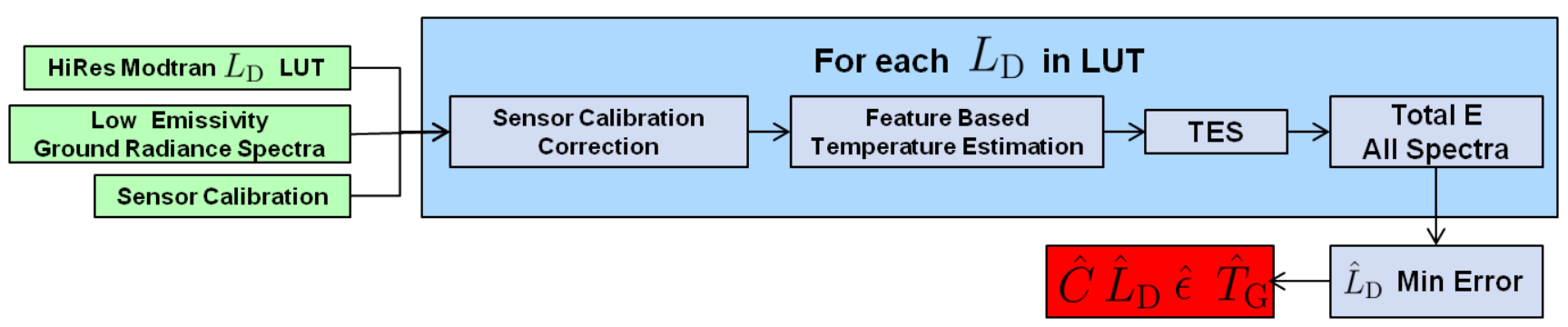

Figure 6. Downwelling radiance estimation pipeline

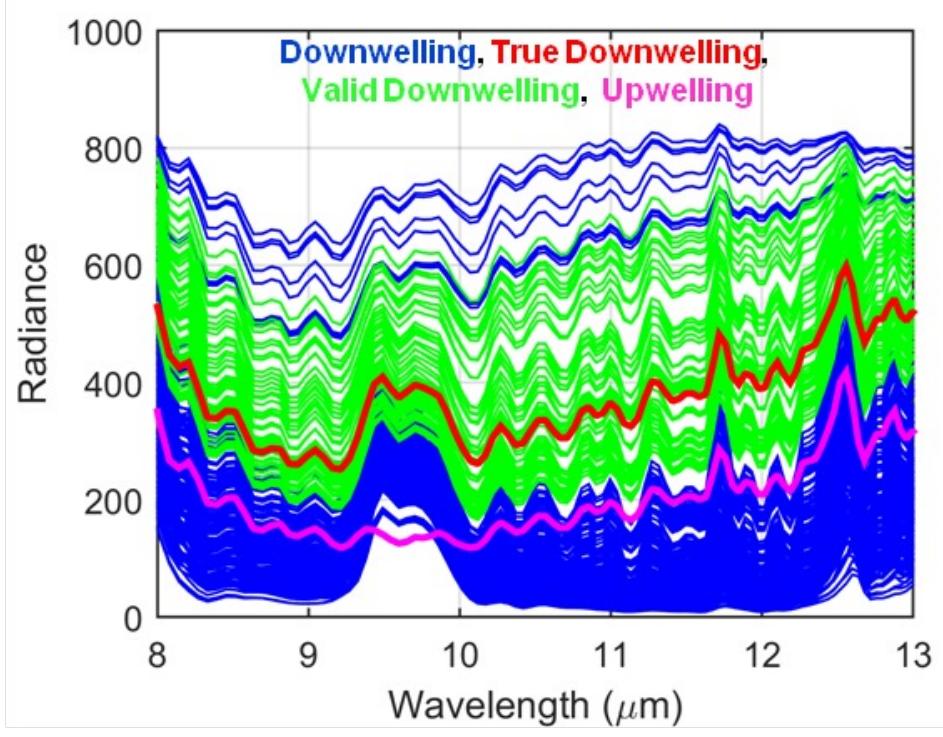

Figure 7. Downsampled Downwelling Radiance MODTRAN Models

centers, and spectral bandwidth (FWHM). Running MODTRAN with permutations of the atmospheric parameters, a multitude of varying downwelling radiance atmospheric models are generated to create a high resolution look-up-table (LUT). Using the wavelength centers and FWHM of the sensor, the high resolution MODTRAN models can be downsampled to the sensor resolution. Fig. 7 shows a LUT consisting of 265 downwelling radiance models that were downsampled to the 128 band SEBASS sensor resolution.

Each of the downwelling radiance models in the LUT is used for TES on the reflective pixel spectra. The $L_{\mathrm{D}}$ model that provides the emissivity spectra with the lowest TES error in Eq. 9 is chosen as the downwelling radiance of the scene. TES is extremely susceptible to small mismatch errors between the estimated downwelling radiance and the true downwelling radiance present in a reflected pixel. Even with the correct downwelling radiance model, mismatch can be caused by sensor calibration errors. Any shifting of the wavelength centers and broadening of the bands will cause a slight discrepancy from the MODTRAN downwelling radiance downsampled with the documented calibration parameters. Calibration correction algorithms are required to determine the correct calibration parameters for downsampling each $L_{\mathrm{D}}$ model and minimize the mismatch [3].

\subsection{Utilization of Reflected Gas Features for Ground Temperature Estimation}

An initial temperature estimate for TES is typically found with the ground channel radiance that provides the maximum brightness temperature. The lower this channel emissivity and the further the pixel is from being a blackbody, the further the initialization temperature will be from the true ground temperature. The lower the emissivity of a material in a pixel, the more visible the sharp reflected gas features of the downwelling radiance. The height of these reflected features are dependent on the height of the corresponding features in 


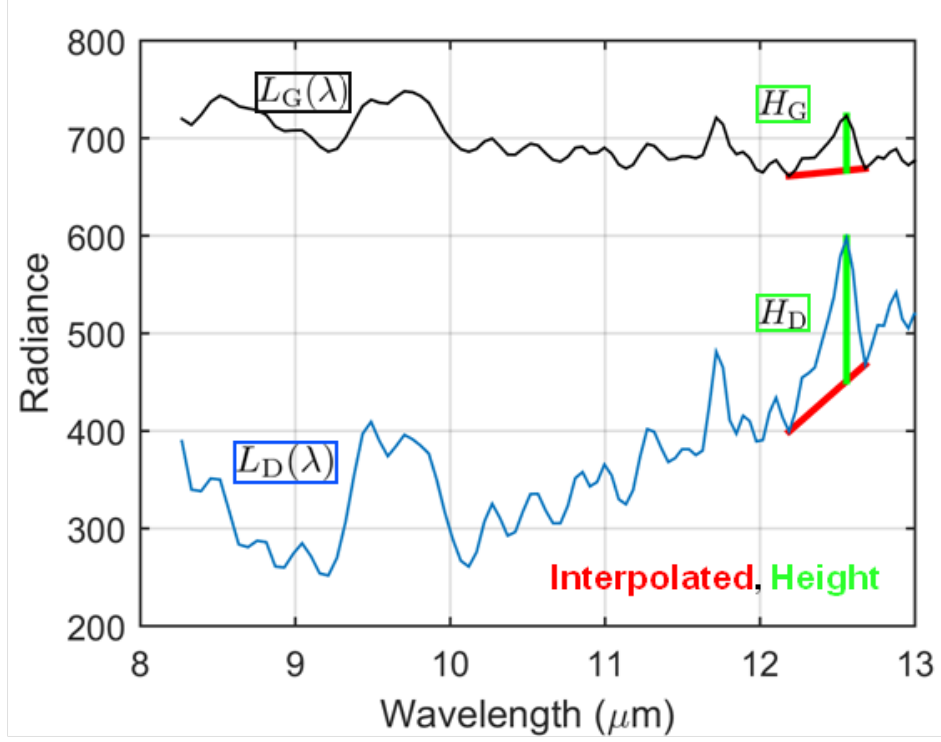

Figure 8. Measured feature height of ground radiance and downwelling radiance

the downwelling radiance and the reflectance $\left[1-\epsilon\left(\lambda_{k}\right)\right]$ across the feature, as shown in Eq. (1). The ground temperature effects the base radiance of the reflected feature, but not the height of the feature.

Since the emissivity is directly related to the height of reflected downwelling features, a large feature can be used to estimate the emissivity at the feature band. The height of a large water feature between $12.2 \mu \mathrm{m}$ and $12.7 \mu \mathrm{m}$ is measured for a ground and downwelling radiance spectra in Fig. 8. The height of the feature is measured from the feature's peak to a line that is linearly interpolated across the base of the feature. The emissivity at a feature band can be estimated by comparing the height of a reflected feature in the ground radiance to a reference height of the same feature from an accurate estimate of the downwelling radiance. Knowing that the height of the $L_{\mathrm{D}}$ reference occurs at an emissivity of 0 , the ratio of the two heights can be used to estimate the emissivity at the feature band $\lambda_{f}$ using Eq. 10.

$$
\hat{\epsilon}\left(\lambda_{f}\right)=1-\frac{H_{\mathrm{G}}}{H_{\mathrm{D}}}
$$

where $H_{\mathrm{G}}$ and $H_{\mathrm{D}}$ are the feature heights of the ground and downwelling radiance respectively. The estimated emissivity is then used to estimate the ground temperature.

$$
\hat{T}_{G_{f}}=B^{-1}\left(\lambda_{f} ; \frac{L_{\mathrm{G}}\left(\lambda_{f}\right)-\left[1-\hat{\epsilon}\left(\lambda_{f}\right)\right] L_{\mathrm{D}}\left(\lambda_{f}\right)}{\hat{\epsilon}\left(\lambda_{f}\right)}\right)
$$

\subsection{Reduction of Possible Downwelling Radiance Models}

The downwelling radiance models present in the LUT are not necessarily all valid models for the available reflective ground radiance spectra. Several techniques are used to remove the invalid downwelling radiance models from the LUT. One technique to filter out invalid models compares the height of a large feature in the downwelling radiance model to the largest feature found in the reflective ground radiance spectra. The feature heights are measured using the technique described in 3.1 to find the improved ground temperature estimate. The downwelling radiance model feature height is the maximum feature height that is reached when a ground radiance spectrum has an emissivity of 0 . Invalid downwelling radiance models have feature heights that are less than the largest feature found in the reflective ground radiance spectra, and would require a negative emissivity to create the observed feature. Such models that require non-physical emissivities are removed from the LUT. 


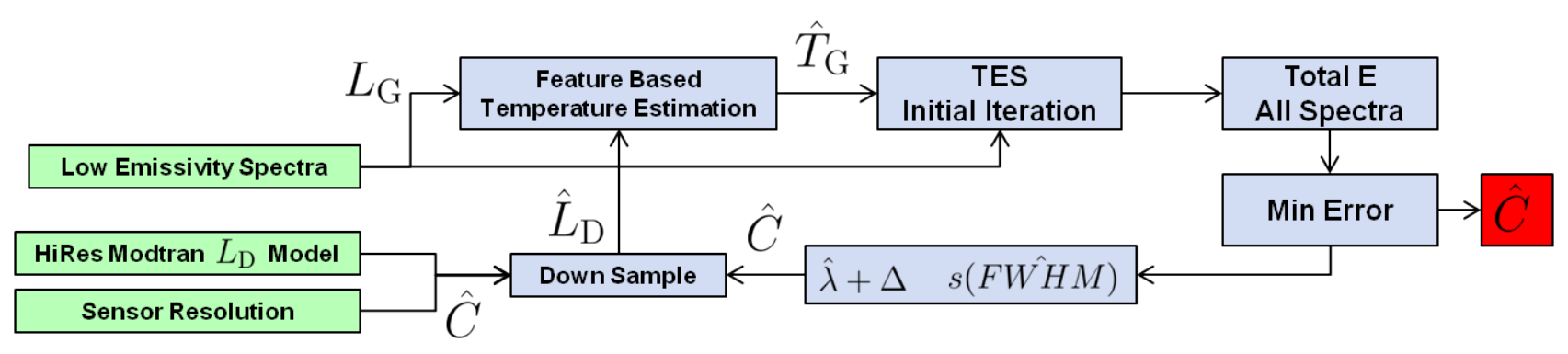

Figure 9. Sensor Calibration Correction Pipeline

The downwelling radiance models in the LUT consist of the entire height of the atmosphere. The upwelling radiance determined by ISAC consists of the portion of atmosphere below the sensor. Since the downwelling radiance is modeled just above the ground where the atmosphere is thickest, and can consist of significantly more atmosphere, it should have a higher radiance. Any downwelling radiance model that has radiances less than the upwelling radiance are removed from the LUT.

The downwelling radiance LUT shown in Fig. 7 includes the true $L_{\mathrm{D}}$ in red and its corresponding $L_{\mathrm{U}}$ at a sensor altitude of $1.5 \mathrm{~km}$ in magenta. Using the simulated ground radiance spectra in Fig. 10, it was possible to reduce the 265 models in the LUT to the 64 green models using the two methods discussed above.

\subsection{Sensor Calibration Correction}

A preflight spectral and radiometric calibration of the hyperspectral sensor is typically accomplished to obtain accurate measurements of band centers $\lambda$ and resolutions FWHM. These measured calibration parameters $C$ can change overtime. Factors such as mechanical vibration and temperature changes can cause spectral shifts $\lambda+\Delta$ and band broadening $s($ FWHM) [6]. The calibration error typically remains constant in the along-track direction but depending on the quality of the sensor can change in the across-track direction[6]. These calibration errors cause a mismatch between the downwelling radiance in the LUT and the reflected downwelling radiance in the ground radiance spectra. This mismatch will prevent TES from completely removing the sharp reflected gas features even when the correct temperature is determined.

The sensor calibration algorithm used to find an accurate estimate of the calibration parameters $\hat{C}=(\lambda+$ $\Delta, s(\mathrm{FWHM}))$ is shown in Fig. 9. Using low emissivity pixel spectra, the current $L_{\mathrm{D}}$, and the documented $C$ as an initial estimate of the calibration, the goal is to find the spectral shift $\Delta$ and broadening factor $s$ that provides the smoothest emissivity spectra and removes the sharp gas features. This process is similar to the technique discussed in [6] that uses reflectance smoothness. Emissivity smoothness is the optimization parameter for TES, so TES is utilized to measure the quality of each $\hat{C}$ iteration. In order to reduce the optimization parameters to just the calibration parameters, TES is done for only one iteration with the feature based temperature estimates. The feature based temperature estimates are close enough to the truth to provide emissivities that are relatively free of reflected atmospheric features when the correct $C$ is found. The temperature estimates are updated each iteration to account for feature changes from the new downsampling. The $\hat{C}$ that provides the lowest overall TES error for all the reflective pixels is chosen as the correct calibration.

A single correction is accomplished for the entire scene, so it is important for the sensor to be of high enough quality for the calibration to remain consistent across-track. Determining a sensor calibration correction for each along-track sample would require several low emissivity pixels in each of the samples [6]. Depending on the scene, there is no guarantee that each sample will contain low emissivity pixels.

\section{DOWNWELLING RADIANCE ESTIMATION SIMULATION}

The proposed techniques used to determine the in-scene downwelling radiance were tested on simulated reflective ground radiance spectra. Simulated spectra were utilized due to the lack of scenes with accurate ground and 
atmospheric truth. Ground radiance spectra were simulated to exclude any effects caused by AC error, and allow for a more precise study of how the downwelling radiance estimate affects TES.

The simulation was done following the flowchart in Fig. 11. A downwelling radiance was chosen from the LUT and downsampled to the 128 band SEBASS sensor calibration $C[2]$. The downsampled downwelling radiance $L_{\mathrm{D}}$ that was used is the red spectrum in Fig. 7 , and the corresponding $L_{\mathrm{U}}$ at $1.5 \mathrm{~km}$ is the magenta spectrum. Three low emissivity spectra were also downsampled to $C$, and are shown in 10a. Five ground radiance spectra were then simulated for each emissivity spectrum. The five spectra for each emissivity were simulated at 5 ground temperatures $T_{\mathrm{G}}$ from $285 \mathrm{~K}-305 \mathrm{~K}$ in $5 \mathrm{~K}$ steps using Eq. 1 . The 15 simulated ground radiance spectra are shown in Fig. 10b, along with the corresponding blackbody spectra in blue. Sensor noise could then be added to the simulated ground radiance spectra by adding white gaussian noise to each spectral band. Calibration error could be added so that the initial calibration estimate is $C_{\mathrm{e}}=(\lambda+\Delta, s(\mathrm{FWHM}))$. The simulated ground radiances, high resolution $L_{\mathrm{D}} \mathrm{LUT}$, and $C_{e}$ are fed into the downwelling radiance estimation algorithm. The downwelling radiance estimation algorithm supplies an estimate of the downwelling radiance $\hat{L}_{\mathrm{D}}$ from the LUT, that is downsampled to a corrected sensor calibration $\hat{C}$. For each of the supplied $L_{\mathrm{G}}$ spectra there are corresponding ground temperature $\hat{T}_{\mathrm{G}}$ and emissivity spectrum $\hat{\epsilon}$ estimates. These estimates are compared to the supplied parameters to determine their quality.

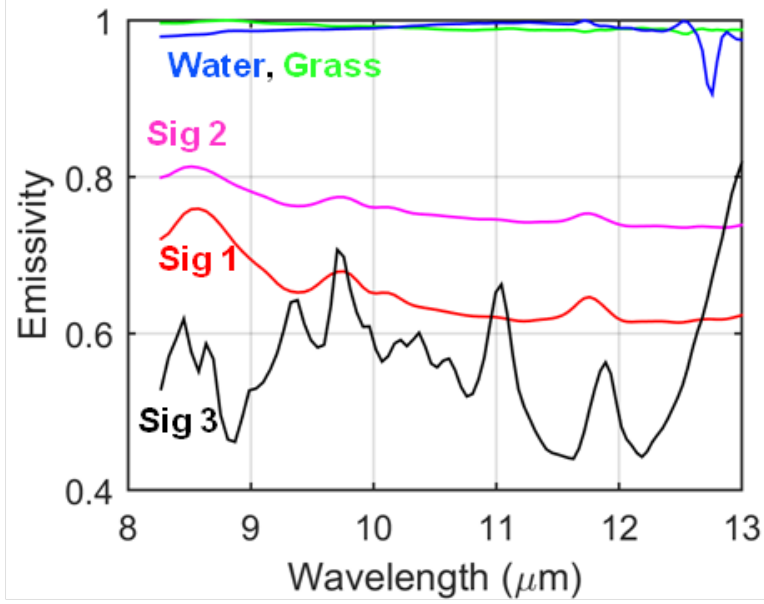

(a) Emissivity Spectra

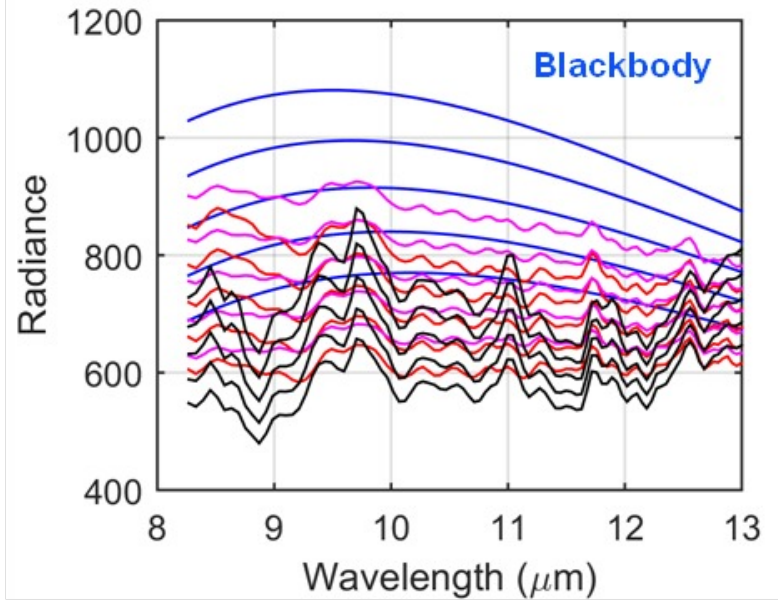

(b) Simulated Ground Radiance and Blackbody Spectra

Figure 10. Emissivity spectra and simulated ground radiance spectra using corresponding low emissivity spectra.

The testing was done in three stages. The first stage used noiseless ground radiance spectra and included no calibration error so that $C=C_{\mathrm{e}}$. The purpose of this stage was to study how the different $L_{\mathrm{D}}$ models effect TES, and whether or not the correct $L_{\mathrm{D}}$ model could be found with no sources of error. Since the two calibrations perfectly matched up, the sensor calibration correction algorithm was removed from the downwelling radiance estimation algorithm, and the hires downwelling radiance models were simply downsampled to $C$. The noiseless ground radiance spectra in Fig. 10 were then processed using the simplified downwelling radiance estimation algorithm that ran TES on each of the signatures using each downsampled $L_{\mathrm{D}}$ in the LUT. The $L_{\mathrm{D}}$ model that provided the lowest overall TES error for the signatures was then chosen.

Errors were added incrementally, to understand how individual errors effected the downwelling estimation process. The second stage was identical to the first except that calibration error was added, and the hires downwelling radiance models were downsampled to $C_{\mathrm{e}}$. The calibration error consisted of a spectral shift of $0.25 \times$ the minimum distance between band centers and a band broadening of $1.1 \times$. The effects of calibration error and $L_{\mathrm{D}}$ mismatch on TES and determining the correct model were studied. The next stage re-inserted the sensor calibration correction algorithm into the downwelling estimation algorithm to correct for the mismatch that was added by $C_{\mathrm{e}}$. The quality of the sensor calibration correction to minimize the $L_{\mathrm{D}}$ mismatch and 


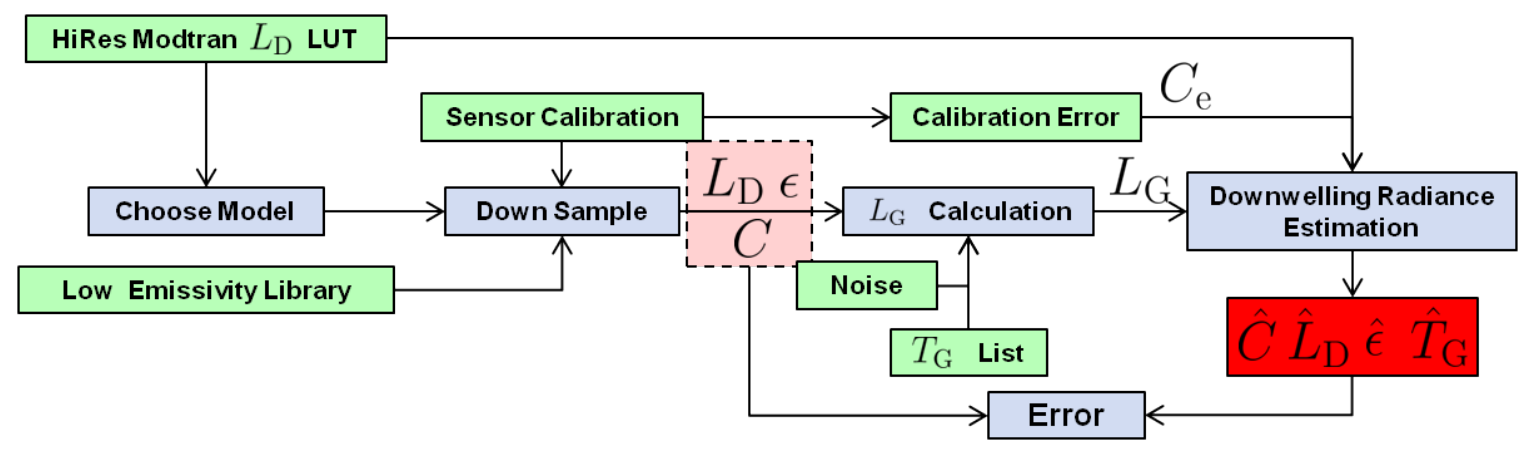

Figure 11. Simulation Pipeline

improve the downwelling radiance estimation and TES was studied. The last stage added sensor noise with a noise equivalent spectral radiance (NESR) of 1 microflick to each band of the ground radiance spectra to determine how noise effected the entire process.

\section{RESULTS}

The four incremental simulation stages were as follows:

- Matched - the $L_{\mathrm{D}}$ used to simulate the ground radiance spectra and the $L_{\mathrm{D}}$ in the LUT were downsampled using the same $C$ so that $C=C_{\mathrm{e}}$.

- Mismatched Calibration - the $L_{\mathrm{D}}$ used to simulate the ground radiance spectra was downsampled at $C$, the $L_{\mathrm{D}}$ in the LUT were downsampled at $C_{\mathrm{e}}=(\lambda+\Delta, s(\mathrm{FWHM})) . \Delta$ was $0.25 \times$ the minimum distance between band centers and the band broadening of $s$ was $1.1 \times$.

- Corrected Calibration - the sensor calibration correction algorithm was used to correct the mismatched $C_{\mathrm{e}}$ used to downsample the $L_{\mathrm{D}}$ LUT.

- Ground Radiance Noise - NESR of 1 microflick was added to each band of the simulated ground radiance spectra.

The results of each stage were compared to each other and the truth using three methods. The true $L_{\mathrm{D}}$ model spectra and the models spectra with lower TES errors are compared in Section 5.1. The TES results using the corresponding low TES error $L_{\mathrm{D}}$ models are studied in the next two sections. The temperature estimates are discussed in section 5.2 and the corresponding emissivity estimates are discussed in section 5.3.

\subsection{Downwelling Radiance Estimates}

For each of the stages the best $L_{\mathrm{D}}$ model found in the LUT with the lowest overall TES error was not the true model used to simulate the ground radiance spectra. Table 1 shows the number of models found that had TES errors less than or equal to the true model. It can be seen that as the stages progressed the number of models decreased. The TES errors for the best and true models of each stage are shown in Table 2. As expected, the TES error was lowest when the downsampling of the LUT matched the ground radiance spectra. The TES error increased when calibration error was included, and was decreased when the sensor calibration was corrected. The sensor calibration compensated for approximately $2 / 3$ of the error caused by the calibration error. When noise was added to the ground radiance spectra, the TES error found with Eq. 9 increased by approximately 800 for the best and true models.

The best $\hat{L}_{\mathrm{D}}$ model spectra for each of the stages are shown in Fig. 12. The best $\hat{L}_{\mathrm{D}}$ spectra are green and the true $\hat{L}_{\mathrm{D}}$ spectra corresponding to the true $L_{\mathrm{D}}$ model in the LUT are red. The rest of the models better than the true $\hat{L}_{\mathrm{D}}$ are blue. The $L_{\mathrm{D}}$ spectra in Fig. 12a for the matching calibration case are quite similar, which explains the small range of TES errors. Table 3 and 4 show the angular and distance error between the $\hat{L}_{\mathrm{D}}$ 


\begin{tabular}{|l|c|c|c|c|}
\hline & Matched & Mismatched & Corrected & Noise \\
\hline \# of Models & 14 & 8 & 6 & 7 \\
\hline
\end{tabular}

Table 1. Number of $L_{\mathrm{D}}$ models better than true model for each stage.

\begin{tabular}{|c|c|c|c|c|}
\hline & Matched & Mismatched & Corrected & Noise \\
\hline Best & 8008.2 & 11560 & 9219.7 & 10040 \\
\hline True & 8020.6 & 11669 & 9261.6 & 10079 \\
\hline Range & 12.4 & 109 & 41.9 & 39 \\
\hline
\end{tabular}

Table 2. TES error for the estimated best and true $L_{\mathrm{D}}$ models for each stage.

spectra and the true $L_{\mathrm{D}}$ for each of the stages. Both errors are zero for the true $\hat{L}_{\mathrm{D}}$ because they are identical with the matching calibration. The max angle and distances errors are quite low.

When calibration error was added, $\hat{L}_{\mathrm{D}}$ spectra significantly different from $L_{\mathrm{D}}$ were found. The best miscalibrated downsampled $\hat{L}_{\mathrm{D}}$ spectra are shown in Fig. 12b. The number of models decreased to 8 . Of the 8 models, only the last two models were consistent between the matching and mismatched calibration models. The angular and distance errors between $\hat{L}_{\mathrm{D}}$ and $L_{\mathrm{D}}$ increased significantly. Sensor calibration correction removed the two highest radiance models from the mis-calibration models. The remaining $\hat{L}_{\mathrm{D}}$ models can be seen in Fig. $12 \mathrm{c}$.

The angular and distance errors between $\hat{L}_{\mathrm{D}}$ and $L_{\mathrm{D}}$ decreased close to their original values. Adding sensor noise to the ground radiances had little effect on the $\hat{L}_{\mathrm{D}}$ spectra as shown in Fig. $12 \mathrm{~d}$.

\begin{tabular}{|c|c|c|c|c|}
\hline & Matched & Mismatched & Corrected & Noise \\
\hline Best & 0.86 & 4.58 & 2.74 & 2.74 \\
\hline True & 0 & 1.37 & 0.17 & 0.14 \\
\hline Max & 1.27 & 5.76 & 2.74 & 4.21 \\
\hline
\end{tabular}

Table 3. Angular Error (Degrees) between the true $L_{\mathrm{D}}$ model and the estimated best and true models for each stage.

\subsection{Ground Temperature Estimates}

The temperature estimate errors for each of the stages are shown in Fig. 13. Each of the figures shows the temperature estimate errors for the $\hat{L}_{\mathrm{D}}$ models corresponding to each stage and all the ground radiance spectra. The ground radiance spectra are grouped into 3 sets of 5 by their emissivities, where each set has temperatures $285 \mathrm{~K}-305 \mathrm{~K}$ in $5 \mathrm{~K}$ steps. Similar to the $\hat{L}_{\mathrm{D}}$ model spectra, the temperature errors corresponding to the best $\hat{L}_{\mathrm{D}}$ spectra are green and red for those corresponding to the true $\hat{L}_{\mathrm{D}}$. The rest of the models in-between are blue. The magenta lines are the initial ground temperature estimates using the maximum brightness temperature of the ground radiance. The black lines are the initial temperature estimates for the true $\hat{L}_{\mathrm{D}}$ model using the method discussed in section 3.1 that compares the height of reflected gas features to the feature in the $\hat{L}_{\mathrm{D}}$ model. The average temperature errors of the 5 spectra for each emissivity spectrum are shown in Table 5 for the best and true $\hat{L}_{\mathrm{D}}$ models.

For all the stages there was a pattern where the temperature error increased with the true temperature of the ground radiance spectrum. The temperature errors for the matching calibration case are shown in Fig. 13a. Due to the similarity of the $\hat{L}_{\mathrm{D}}$ spectra, the temperature errors for each $\hat{L}_{\mathrm{D}}$ model were nearly identical. The temperature errors were always under $1.5 \mathrm{~K}$ with averages less than $0.5 \mathrm{~K}$. Ground radiance spectra simulated with Sig 2 , the highest emissivity spectrum, had the lowest error. The feature based initial temperature estimates remained below $2 \mathrm{~K}$ and were significantly better than the estimates using the maximum brightness temperature. The temperature error increased significantly when the calibration error was added, as shown in Fig. 13b. The average errors in Table 5 increased by over an order of magnitude for the true model. The feature based initial temperature estimates still remained below $2 \mathrm{~K}$, and were better than the final estimates. The sensor calibration correction was able to correct the temperature estimates with errors within $-0.5 \mathrm{~K}$ for the true model, where all the estimates were too low. The feature based initial temperature estimates were nearly identical to the initial 


\begin{tabular}{|c|c|c|c|c|}
\hline & Matched & Mismatched & Corrected & Noise \\
\hline Best & 193.05 & 762.93 & 467.87 & 467.93 \\
\hline True & 0 & 76.76 & 9.60 & 7.70 \\
\hline Max & 217.25 & 1034.2 & 467.87 & 754.23 \\
\hline
\end{tabular}

Table 4. Distance Error between the true $L_{\mathrm{D}}$ model and the estimated best and true models for each stage.

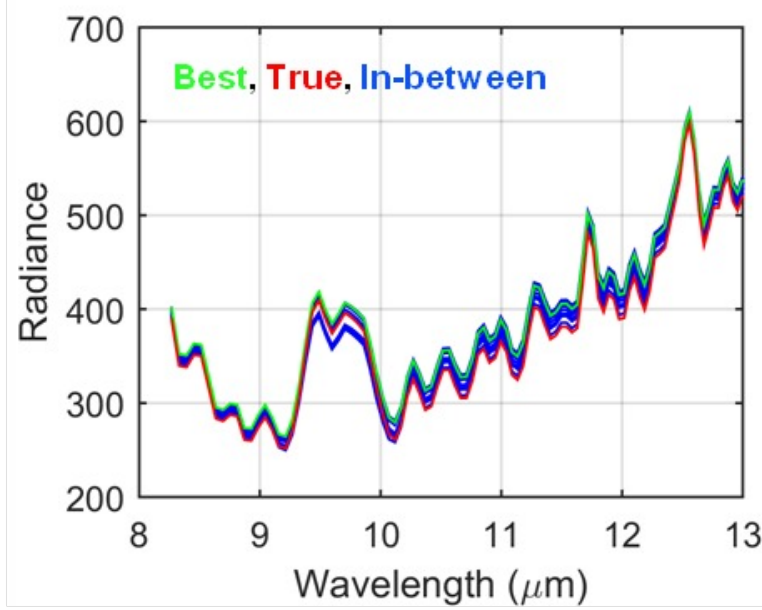

(a) Matching Calibration

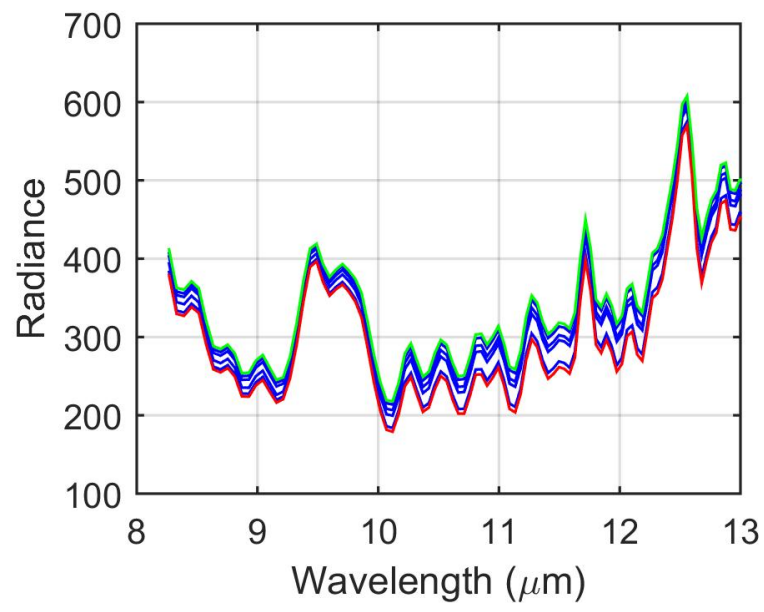

(c) Corrected Calibration

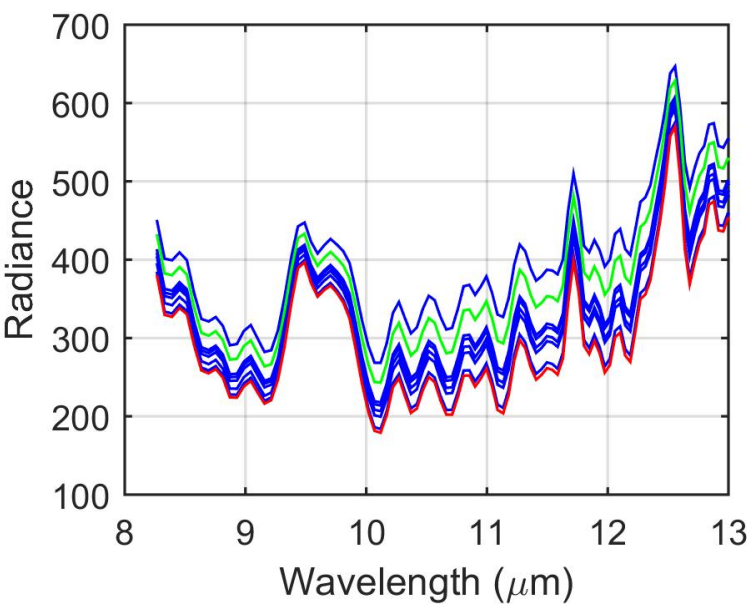

(b) Mismatched Calibration

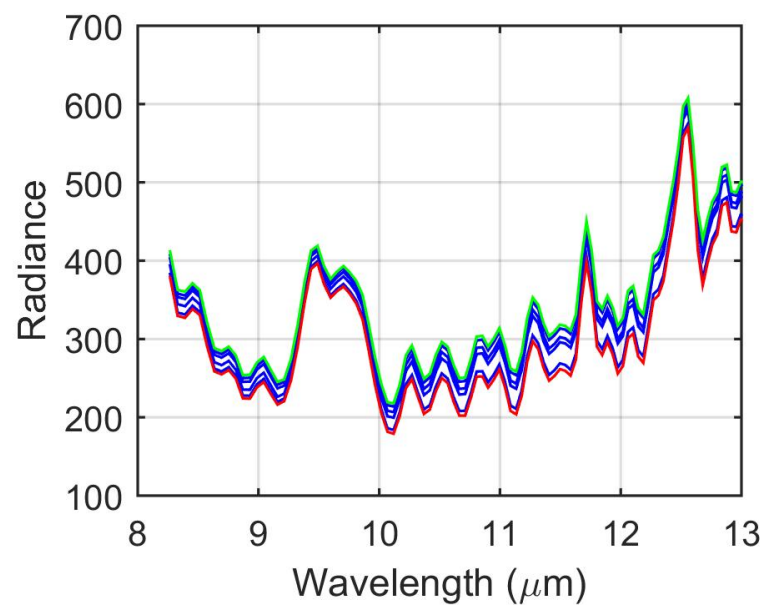

(d) Noisy Ground Radiance Corrected Calibration

Figure 12. Best $L_{\mathrm{D}}$ models.

estimates for the matching calibration. Sensor noise had little effect on the temperature error other than adding a slight randomness.

\subsection{Emissivity Estimates}

The emissivity estimates with the best $\hat{L}_{\mathrm{D}}$ for each of the stages are shown in Fig. 14 . The three truth emissivity spectra are red, and the estimates are blue. The average angular and distance emissivity errors of the 5 spectra for each emissivity spectrum are shown in Tables 6 and 7 for the best and true $\hat{L}_{\mathrm{D}}$ models. The emissivity estimates for the matching calibration in Fig. 14a are below the truth and have a slightly different truth. The temperatures were as close to the truth as the true $\hat{L}_{\mathrm{D}}$, so the difference in emissivity is caused by the slight shape and height difference between the best $\hat{L}_{\mathrm{D}}$ and $L_{\mathrm{D}}$. The effects of mismatched calibration are evident in 


\begin{tabular}{|l|c|c|c|c|c|c|c|c|c|c|c|c|}
\hline & \multicolumn{3}{|c|}{ Matched } & \multicolumn{3}{c|}{ Mismatched } & \multicolumn{3}{c|}{ Corrected } & \multicolumn{3}{c|}{ Noise } \\
\hline & Sig 1 & Sig 2 & Sig3 & Sig 1 & Sig 2 & Sig 3 & Sig 1 & Sig 2 & Sig 3 & Sig 1 & Sig 2 & Sig 3 \\
\hline Best & 0.37 & 0.11 & 0.43 & 1.25 & 0.56 & 3.11 & -1.52 & -0.98 & -1.66 & -1.53 & -0.82 & -1.47 \\
\hline True & 0.35 & 0.10 & 0.41 & 3.67 & 1.95 & 5.91 & -0.46 & -0.36 & -0.34 & -0.34 & -0.12 & -0.01 \\
\hline
\end{tabular}

Table 5. Average Temperature Error (K)

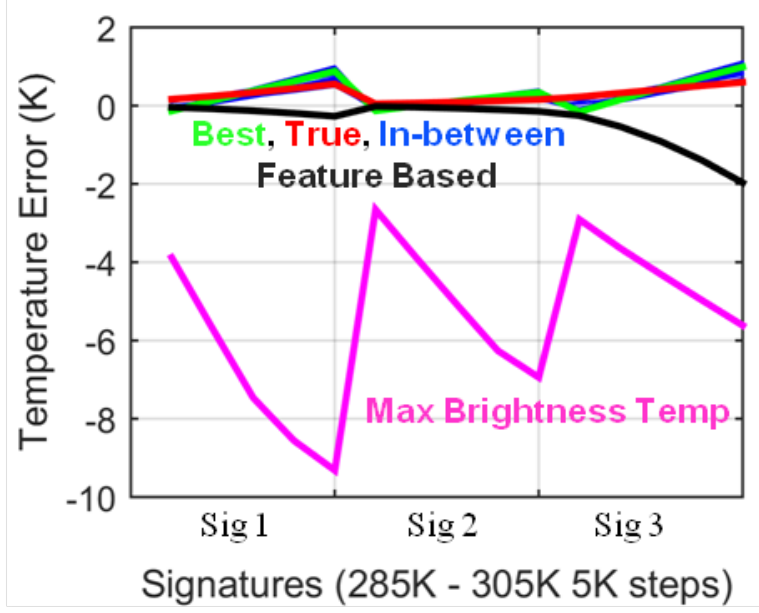

(a) Matching Calibration

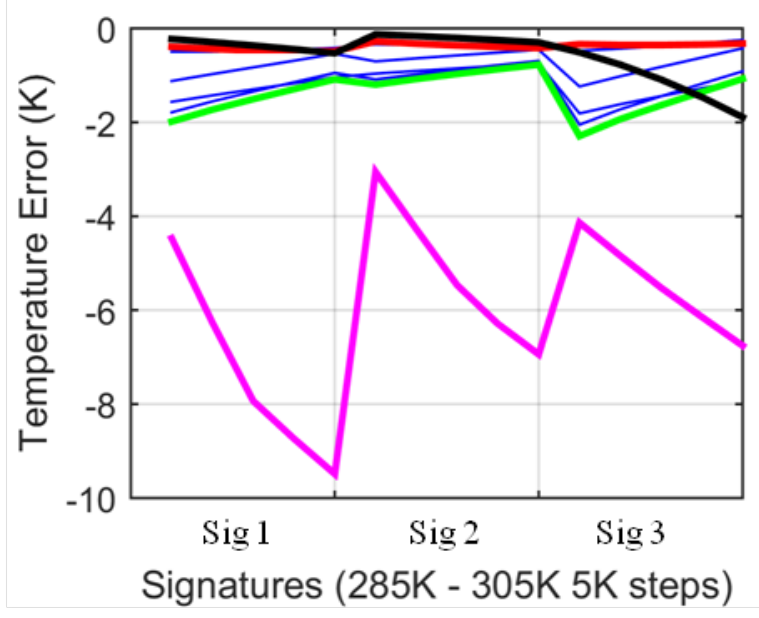

(c) Corrected Calibration

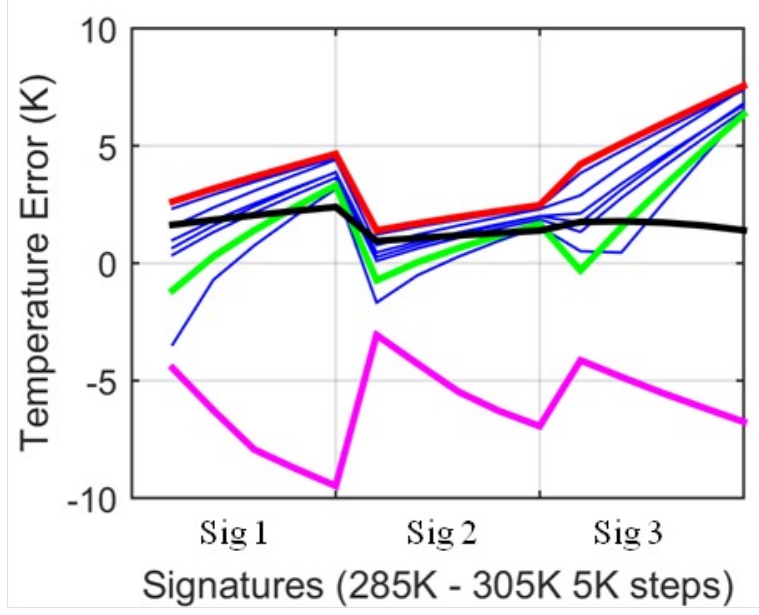

(b) Mismatched Calibration

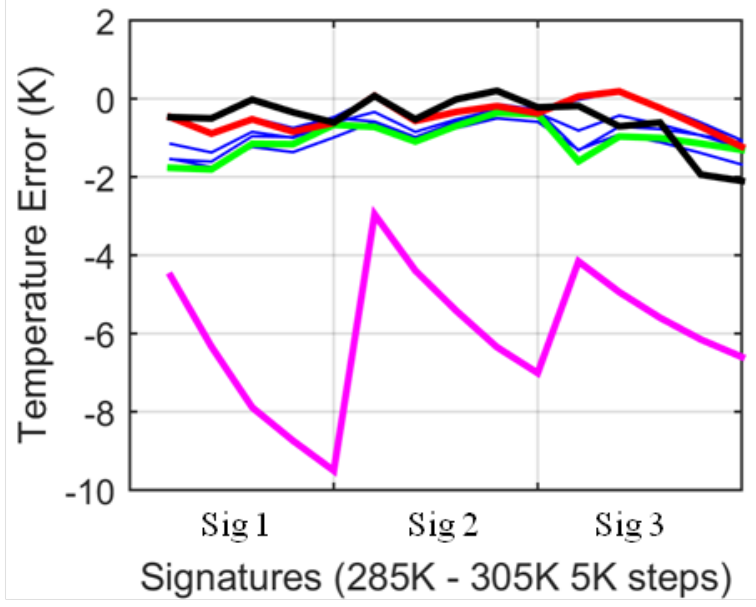

(d) Noisy Ground Radiance Corrected Calibration

Figure 13. Ground temperature estimates of ground radiance spectra with corresponding best $L_{\mathrm{D}}$ models.

Fig. 14b from the roughness of the emissivity spectra. The temperature estimates and $\hat{L}_{\mathrm{D}}$ were above the truth lowering the emissivity estimates. The sensor calibration was able to correct the $L_{\mathrm{D}}$ mismatch and give the emissivity estimates in Fig. 14c that were close to the matching calibration estimates. The sensor noise added to the ground radiance spectra was transfered to the emissivity estimates in Fig. 14d, but the overall shapes remained the same.

The emissivity estimates with the true $\hat{L}_{\mathrm{D}}$ for each of the stages are shown in Fig. 15 . With the true $\hat{L}_{\mathrm{D}}$, the emissivity estimates are significantly closer to the truth. Mismatched calibration added roughness to the emissivity estimates shown in Fig. 15b. TES failed giving temperature estimates several degrees above the truth which lowered the emissivity estimates. The sensor calibration was able to correct the $L_{\mathrm{D}}$ mismatch and give 
the emissivity estimates in Fig. 15c that were close to the matching calibration estimates. The estimates were slightly above the truth due to temperature estimates slightly below the truth. The sensor noise added to the ground radiance spectra was transfered to the emissivity estimates in Fig. 15d, but the overall shapes remained the same.

The optimum emissivity estimates with the true $\hat{L}_{\mathrm{D}}$ models of each stage were determined by calculating the emissivities with the correct temperatures of each ground radiance spectrum and the true $\hat{L}_{\mathrm{D}}$ model. By calculating the emissivities with the correct temperature, the emissivity estimates are free of TES errors. The calculated emissivities for all the stages excluding the matching calibration are shown in Fig. 16. Fig. 16a show the true $\hat{L}_{\mathrm{D}}$ models for each of the stages. Except for the mismatched calibration case in Fig. 16b were TES failed, the estimated and calculated emissivities are close to each other. The average angular and distance emissivity errors of the 5 calculated emissivity spectra for each emissivity spectrum are shown in Tables 6 and 7 .

\begin{tabular}{|c|c|c|c|c|c|c|c|c|c|c|c|c|}
\hline & \multicolumn{3}{|c|}{ Matched } & \multicolumn{3}{c|}{ Mismatched } & \multicolumn{3}{c|}{ Corrected Noise } \\
\hline & Sig 1 & Sig 2 & Sig3 & Sig 1 & Sig 2 & Sig 3 & Sig 1 & Sig 2 & Sig 3 & Sig 1 & Sig 2 & Sig 3 \\
\hline Best & 0.89 & 0.47 & 1.23 & 4.04 & 2.14 & 5.64 & 1.83 & 0.97 & 2.70 & 1.85 & 1.01 & 2.71 \\
\hline True & 0.11 & 0.03 & 0.14 & 1.09 & 0.62 & 1.61 & 0.15 & 0.11 & 0.13 & 0.21 & 0.16 & 0.21 \\
\hline Correct Temp & - & - & - & 0.66 & 0.39 & 0.76 & 0.08 & 0.05 & 0.09 & 0.18 & 0.15 & 0.19 \\
\hline
\end{tabular}

Table 6. Average Angular Emissivity Error (Degrees)

\begin{tabular}{|c|c|c|c|c|c|c|c|c|c|c|c|c|}
\hline & \multicolumn{3}{|c|}{ Matched } & \multicolumn{3}{c|}{ Mismatched } & \multicolumn{3}{c|}{ Corrected } & \multicolumn{3}{c|}{ Noise } \\
\hline & Sig 1 & Sig 2 & Sig3 & Sig 1 & Sig 2 & Sig 3 & Sig 1 & Sig 2 & Sig 3 & Sig 1 & Sig 2 & Sig 3 \\
\hline Best & 0.25 & 0.15 & 0.28 & 0.92 & 0.60 & 1.22 & 0.22 & 0.13 & 0.30 & 0.22 & 0.15 & 0.32 \\
\hline True & 0.06 & 0.02 & 0.07 & 0.57 & 0.36 & 0.79 & 0.08 & 0.07 & 0.05 & 0.06 & 0.04 & 0.04 \\
\hline Correct Temp & - & - & - & 0.07 & 0.05 & 0.08 & 0.009 & 0.006 & 0.009 & 0.021 & 0.020 & 0.020 \\
\hline
\end{tabular}

Table 7. Average Distance Emissivity Error

\section{SUMMARY AND FUTURE WORK}

It was found that the true $\hat{L}_{\mathrm{D}}$ model did not provide emissivity estimates with the minimum TES error. However, the best $\hat{L}_{\mathrm{D}}$ model was still within $3^{\circ}$ of the $L_{\mathrm{D}}$, and provided temperature estimates within $2 \mathrm{~K}$ and emissivity estimates within $3^{\circ}$ of the truth. It was shown how mismatched calibration could cause TES to fail, but that a majority of the mismatch could be removed with the sensor calibration algorithm returning the results close to the originals. A feature based temperature estimation was shown that provides an improved initialization temperature for TES within $2 \mathrm{~K}$ of the truth, and is resistant to $L_{\mathrm{D}}$ mismatch.

Future work will include developing techniques that can speed up the time of convergence to an accurate $\hat{L}_{\mathrm{D}}$ model. These techniques will include methods to further reduce the number of possible $\hat{L}_{\mathrm{D}}$ models in the LUT, and limiting the number of models that sensor calibration correction is implemented on. Further testing will also be done with varying low emissivity spectra. The largest limitation to this test was the lack of atmospheric compensation error in the ground radiance error. In order to add this error to the model, scenes need to be developed with statistically meaningful classes and atmospheres with a small amount of variability need to be applied. Atmospheric compensation algorithms can then be tested on simulated scenes to determine possible errors and there effects on TES and determining a downwelling radiance model.

\section{References}

[1] Manolakis, D., Marden, D., and Shaw, G., "Hyperspectral image processing for automatic target detection applications," Lincoln Laboratory Journal 14(1), 79-116 (2003).

[2] Young, S. J., Johnson, B. R., and Hackwell, J. A., "An in-scene method for atmospheric compensation of thermal hyperspectral data," Journal of Geophysical Research: Atmospheres 107(D24), ACH 14-1-ACH 14-20 (2002). 4774. 


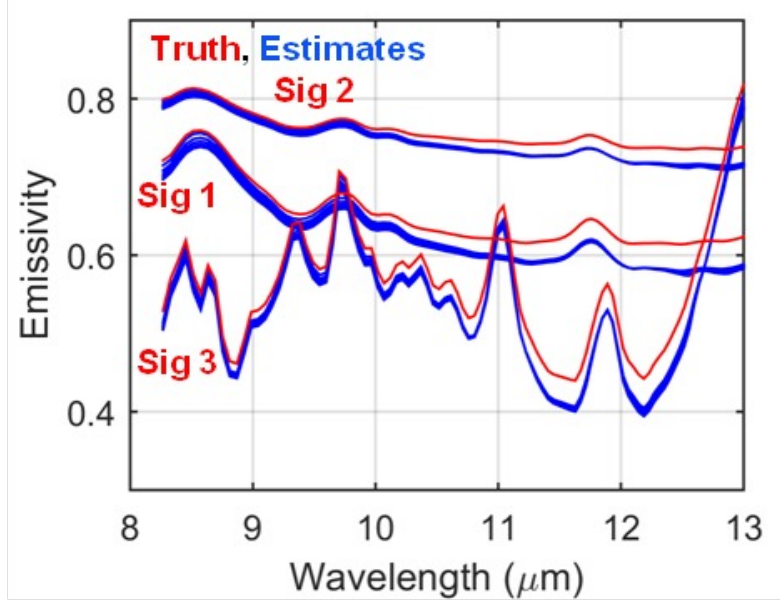

(a) Matching Calibration

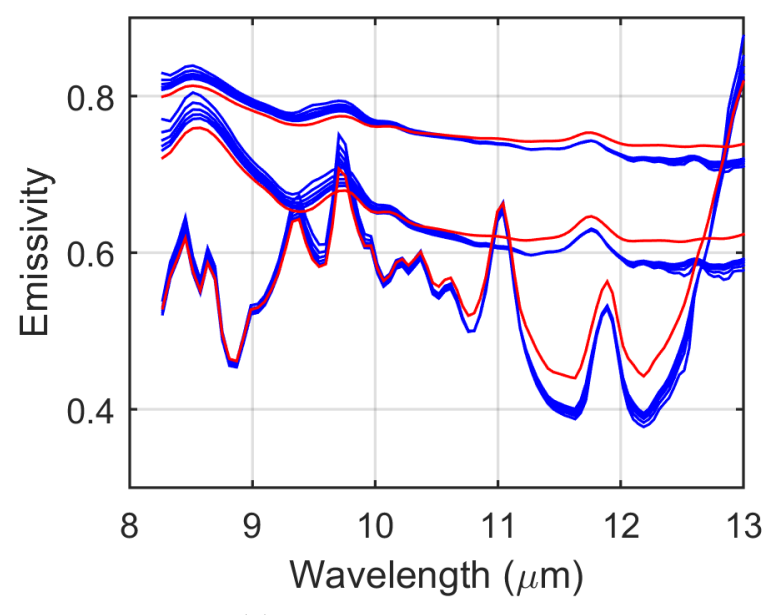

(c) Corrected Calibration

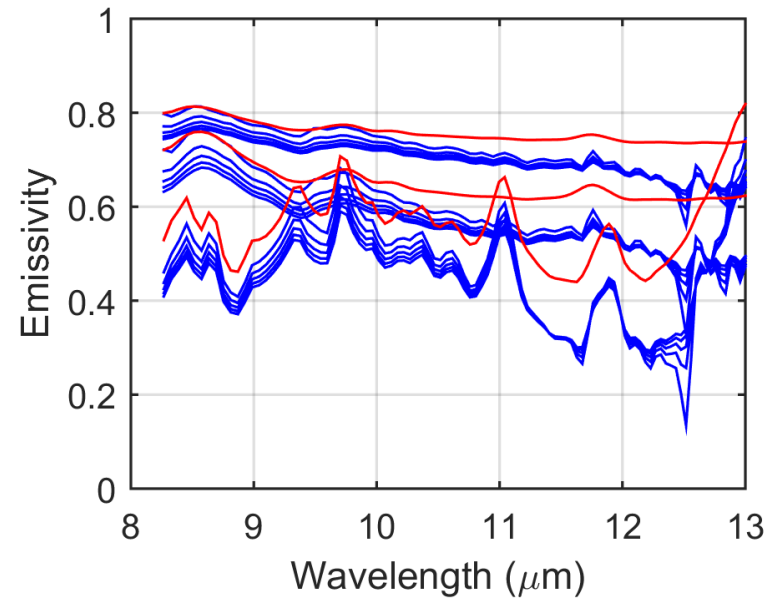

(b) Mismatched Calibration

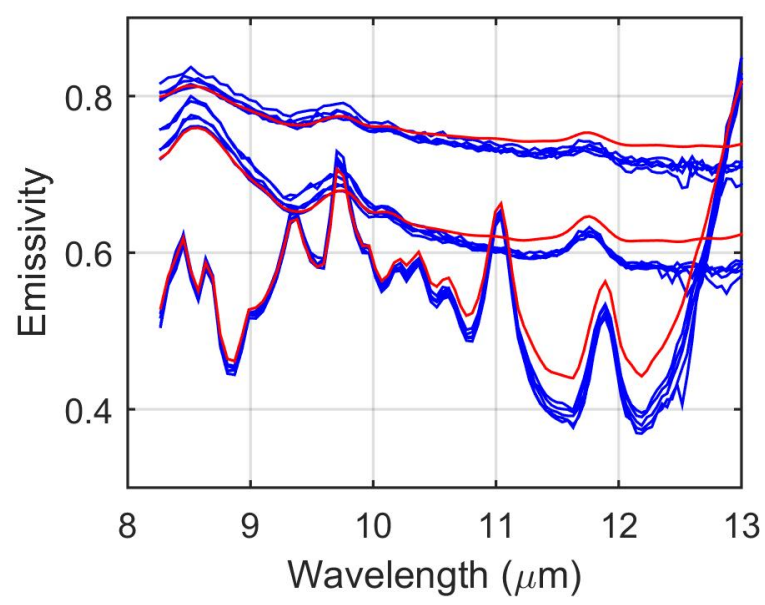

(d) Noisy Ground Radiance Corrected Calibration

Figure 14. Emissivity estimates from ground radiance spectra with best $L_{\mathrm{D}}$ models.

[3] Borel, C., "Error analysis for a temperature and emissivity retrieval algorithm for hyperspectral imaging data," Proc. SPIE 6565, 65651Q-65651Q-12 (2007).

[4] Boonmee, M., Land Surface Temperature and Emissivity Retrieval from Thermal Infrared Hyperspectral Imagery, $\mathrm{PhD}$ thesis (2007).

[5] Berk, A., Anderson, G. P., Acharya, P. K., Bernstein, L. S., Muratov, L., Lee, J., Fox, M., Adler-Golden, S. M., Chetwynd, Jr., J. H., Hoke, M. L., Lockwood, R. B., Gardner, J. A., Cooley, T. W., Borel, C. C., Lewis, P. E., and Shettle, E. P., "Modtran5: 2006 update," (2006).

[6] Guanter, L., Richter, R., and Moreno, J., "Spectral calibration of hyperspectral imagery using atmospheric absorption features," Appl. Opt. 45, 2360-2370 (Apr 2006). 


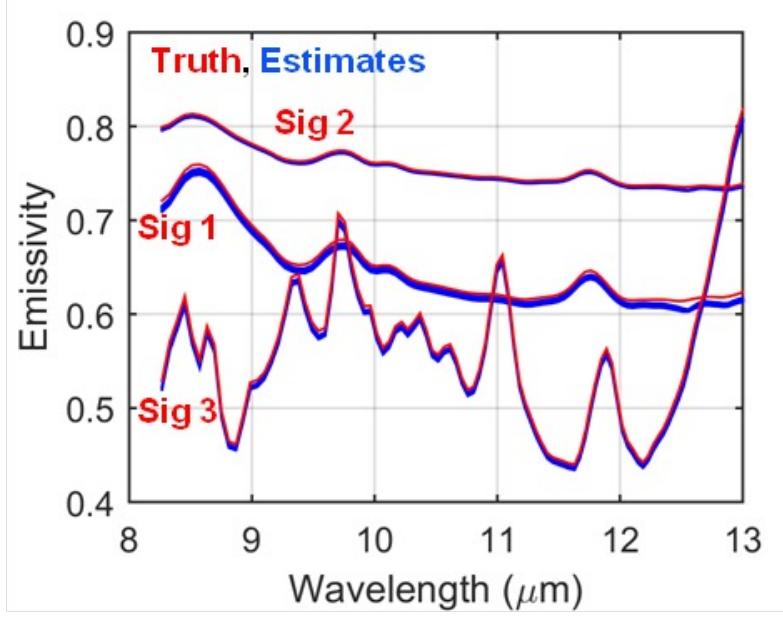

(a) Matching Calibration

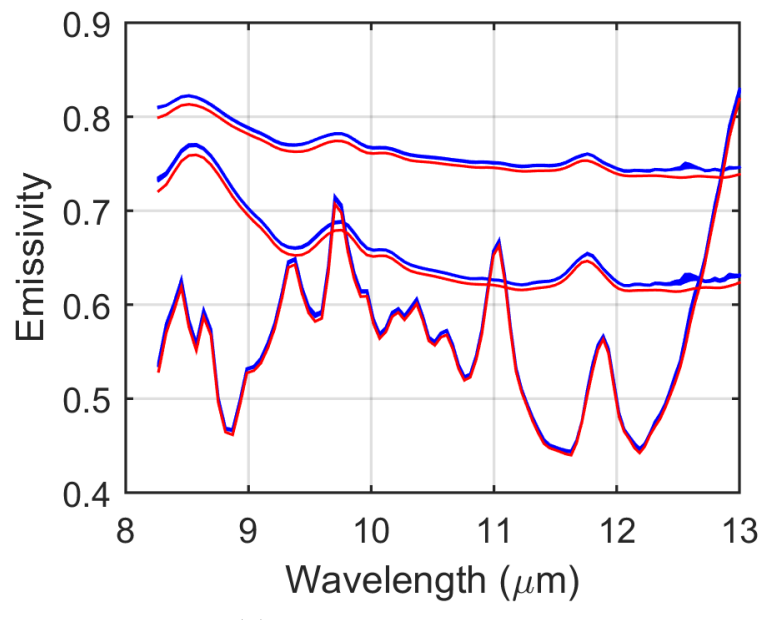

(c) Corrected Calibration

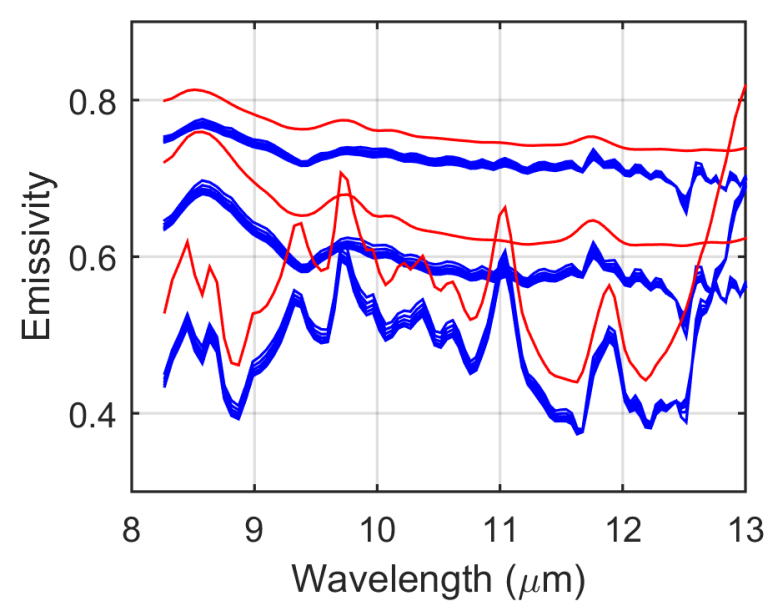

(b) Mismatched Calibration

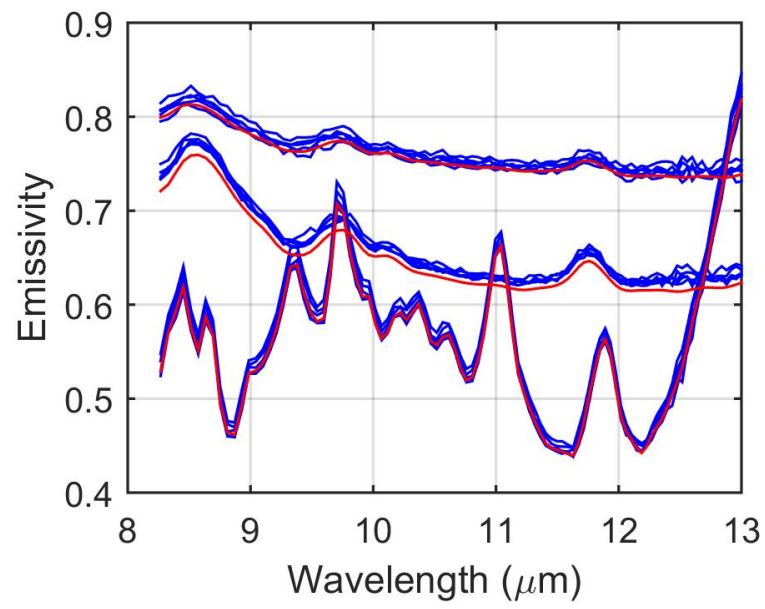

(d) Noisy Ground Radiance Corrected Calibration

Figure 15. Emissivity estimates from ground radiance spectra with true $L_{\mathrm{D}}$ models. 


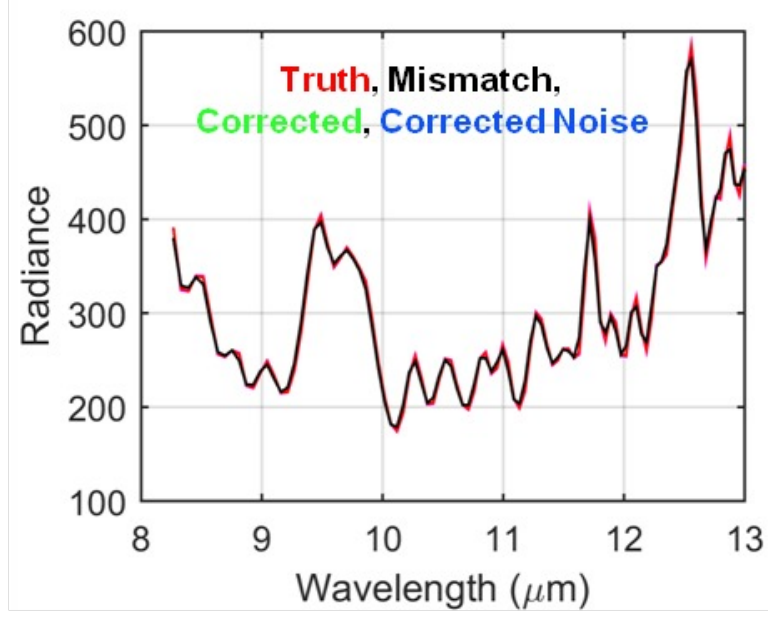

(a) Stages of true $L_{\mathrm{D}}$ model

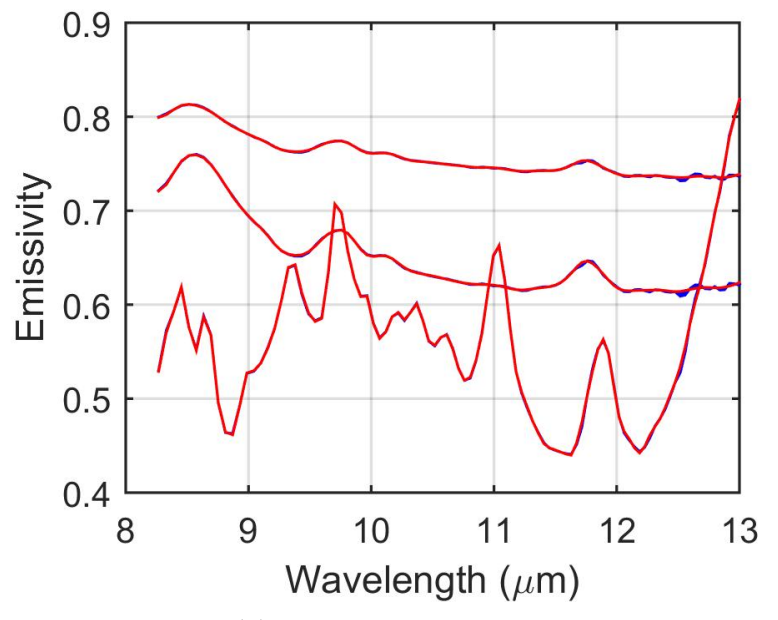

(c) Corrected Calibration

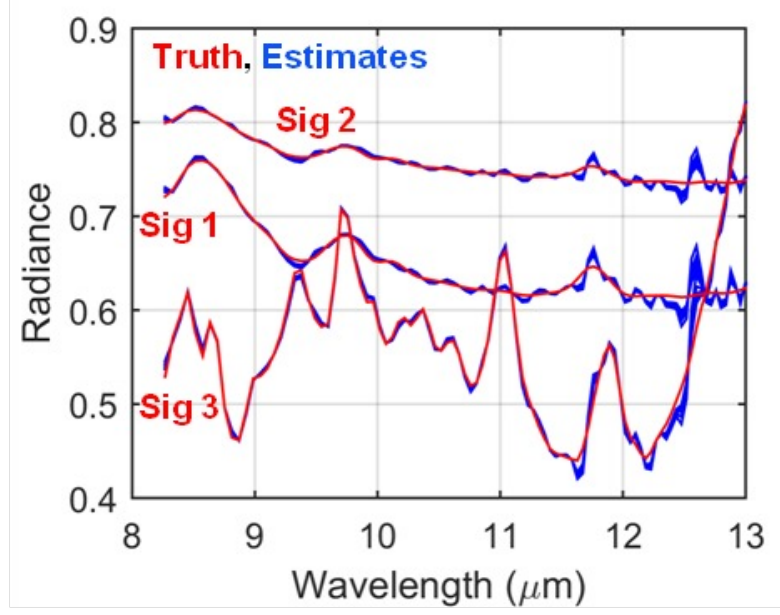

(b) Mismatched Calibration

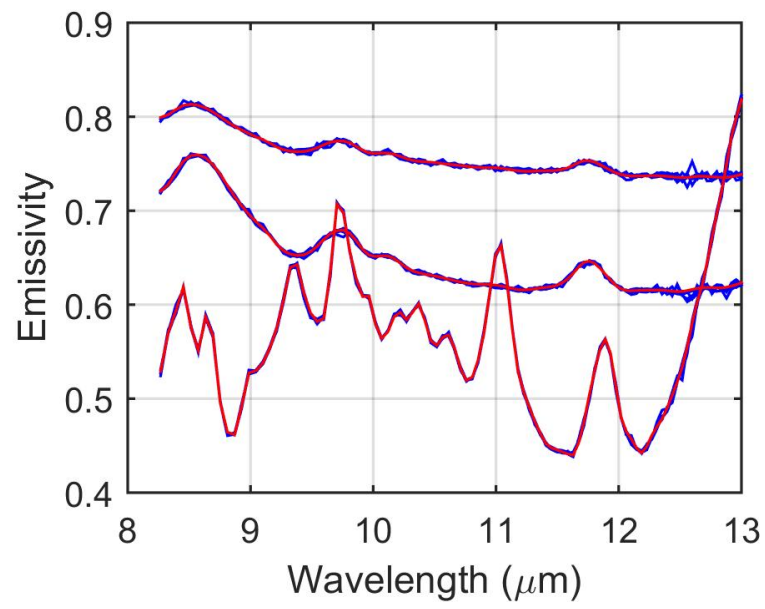

(d) Noisy Ground Radiance Corrected Calibration

Figure 16. Calculated Emissivities using the true ground temperatures and true $L_{\mathrm{D}}$ model of each stage 